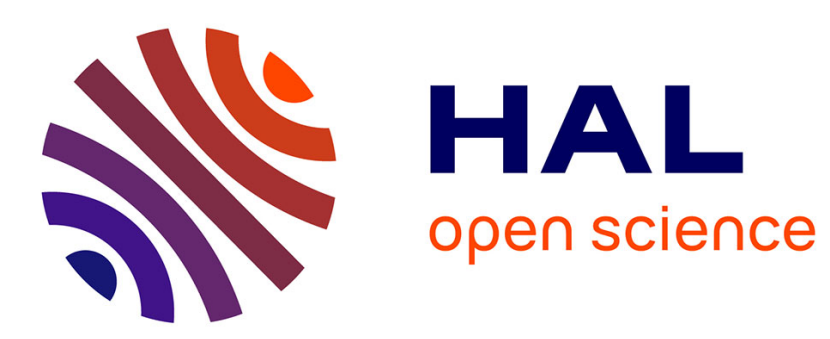

\title{
On the Uniqueness of Global Multiple SLEs
}

Vincent Beffara, Eveliina Peltola, Hao Wu

\section{- To cite this version:}

Vincent Beffara, Eveliina Peltola, Hao Wu. On the Uniqueness of Global Multiple SLEs. Annals of Probability, 2021, 49 (1), pp.400-434. 10.1214/20-AOP1477 . hal-01691513v2

\section{HAL Id: hal-01691513 \\ https://hal.science/hal-01691513v2}

Submitted on 22 Nov 2021

HAL is a multi-disciplinary open access archive for the deposit and dissemination of scientific research documents, whether they are published or not. The documents may come from teaching and research institutions in France or abroad, or from public or private research centers.
L'archive ouverte pluridisciplinaire HAL, est destinée au dépôt et à la diffusion de documents scientifiques de niveau recherche, publiés ou non, émanant des établissements d'enseignement et de recherche français ou étrangers, des laboratoires publics ou privés. 


\title{
ON THE UNIQUENESS OF GLOBAL MULTIPLE SLES
}

\author{
By Vincent Beffara ${ }^{1}$, Evelina Peltola ${ }^{2}$ And HaO Wu ${ }^{3}$ \\ ${ }^{1}$ Institut Fourier, CNRS \& University Grenoble Alpes, vincent.beffara@univ-grenoble-alpes.fr \\ ${ }^{2}$ Institute for Applied Mathematics, University of Bonn, eveliina.peltola@ hcm.uni-bonn.de \\ ${ }^{3}$ Yau Mathematical Sciences Center, Tsinghua University, hao.wu.proba@gmail.com
}

This article focuses on the characterization of global multiple SchrammLoewner evolutions (SLE). The chordal SLE describes the scaling limit of a single interface in various critical lattice models with Dobrushin boundary conditions, and similarly, global multiple SLEs describe scaling limits of collections of interfaces in critical lattice models with alternating boundary conditions. In this article, we give a minimal amount of characterizing properties for the global multiple SLEs: we prove that there exists a unique probability measure on collections of pairwise disjoint continuous simple curves with a certain conditional law property. As a consequence, we obtain the convergence of multiple interfaces in the critical Ising, FK-Ising and percolation models.

1. Introduction. At the turn of the millennium, O. Schramm introduced random fractal curves in the plane which he called "stochastic Loewner evolutions" (SLE) [32, 34], and which have since then been known as Schramm-Loewner evolutions. He proved that these probability measures on curves are the unique ones that enjoy the following two properties: their law is conformally invariant and, viewed as growth processes (via Loewner's theory), they have the domain Markov property - a memorylessness property of the growing curve. These properties are natural from the physics point of view, and in many cases, it has been verified that interfaces in critical planar lattice models of statistical physics converge in the scaling limit to SLE type curves; see [4, 5, 26, 36, 38, 39] for examples.

In the chordal case, there is a one-parameter family $\left(\mathrm{SLE}_{\kappa}\right)$ of such curves, parameterized by a real number $\kappa \geq 0$, which is believed to be related to universality classes of the critical models, as well as to the central charges of the corresponding conformal field theories. In this article, we consider several interacting $\mathrm{SLE}_{\kappa}$ curves, multiple SLEs. We prove in Section 3 that, when $\kappa \in(0,4]$, there exists a unique multiple $\mathrm{SLE}_{\kappa}$ measure on families of curves with a given connectivity pattern, as detailed in Theorem 1.2 below. Such measures have already been considered in many works [3, 8, 13,21,25], but we have not found a conceptual approach in the literature, in terms of a minimal amount of characterizing properties in the spirit of Schramm's classification.

The results on convergence of a single discrete interface to an $\mathrm{SLE}_{\kappa}$ curve in the scaling limit are all rather involved. On the other hand, after the characterization of the multiple SLEs, it is relatively straightforward to extend these convergence results to prove that multiple interfaces also converge to multiple $\mathrm{SLE}_{\kappa}$ curves. Indeed, the relative compactness of the interfaces in a suitable topology can be verified with little effort, for example, using results in $[10,19]$, and the main problem is then to identify the limit (i.e., to prove that the subsequential limits are given by a unique collection of random curves).

As an application, we show that the chordal interfaces in the critical Ising model with alternating boundary conditions converge to the multiple $\mathrm{SLE}_{\kappa}$ with parameter $\kappa=3$, in the 


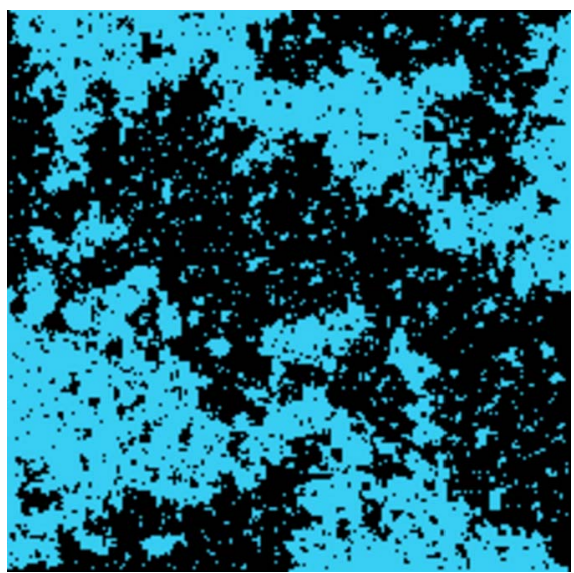

FIG. 1. Simulation of the critical Ising model with alternating boundary conditions. There are eight marked points on the boundary of the polygon $\Omega^{\delta}$ and, therefore, four interfaces connect the marked points pairwise. We only illustrate one possible connectivity of the curves (the reader may verify that there are 14 different topological possibilities).

sense detailed in Proposition 1.3 and Section 4.4. In contrast to the previous work [15] of K. Izyurov, we work with the global collection of curves and condition on the event that the interfaces form a given connectivity pattern; see Figure 1 for an illustration. We also identify the marginal law of one curve in the scaling limit as a weighted chordal $\mathrm{SLE}_{3}$. For the identification of the scaling limit, we use the known convergence of a single critical Ising interface to the chordal $\mathrm{SLE}_{3}$ [5] combined with our characterization of the multiple $\mathrm{SLE}_{3}$, and certain technical estimates to rule out pathological behavior of the curves.

The explicit construction of global multiple $\mathrm{SLE}_{\kappa}$ given in [21, 25, 31], and summarized in Section 3 of the present article, fails for $\kappa>4$. Nevertheless, we discuss in Section 4 how, using information from discrete models, one could extend the classification of the multiple $\mathrm{SLE}_{\kappa}$ (our Theorem 1.2) to the range $\kappa \in(4,6]$. More precisely, we show that the convergence of a single interface in the critical random-cluster model combined with a RussoSeymour-Welsh type (RSW) estimate implies the existence and uniqueness of a global multiple $\mathrm{SLE}_{\kappa}$, where $\kappa \in(4,6]$ is related to the cluster weight $q$ via equation (4.2). In the special case of the FK-Ising model $(q=2)$, thanks to the results of [5, 7, 10, 19, 40], we do obtain the convergence of any number of chordal interfaces to the unique multiple $\mathrm{SLE}_{16 / 3}$; see Proposition 1.4. For general $\kappa \in(4,6)$, this result remains conditional on the convergence of a single interface. The case $\kappa=6$ corresponds to critical percolation, where the convergence also follows by $[4,38]$.

1.1. Global multiple SLEs. Throughout, we let $\Omega \subset \mathbb{C}$ denote a simply connected domain with $2 N$ distinct points $x_{1}, \ldots, x_{2 N} \in \partial \Omega$ appearing in counterclockwise order along the boundary on locally connected boundary segments. We call the $(2 N+1)$ tuple $\left(\Omega ; x_{1}, \ldots, x_{2 N}\right)$ a (topological) polygon. We consider curves in $\Omega$ each of which connects two points among $\left\{x_{1}, \ldots, x_{2 N}\right\}$. These curves can have various planar (i.e., noncrossing) connectivities. We describe the connectivities by planar pair partitions $\alpha=$ $\left\{\left\{a_{1}, b_{1}\right\}, \ldots,\left\{a_{N}, b_{N}\right\}\right\}$, where $\left\{a_{1}, b_{1}, \ldots, a_{N}, b_{N}\right\}=\{1,2, \ldots, 2 N\}$. We call such $\alpha$ (planar) link patterns and denote the set of them by $\mathrm{LP}_{N}$, for each $N \geq 1$. Given a link pattern $\alpha \in \mathrm{LP}_{N}$ and $\{a, b\} \in \alpha$, we denote by $\alpha /\{a, b\}$ the link pattern in $\mathrm{LP}_{N-1}$ obtained by removing $\{a, b\}$ from $\alpha$ and then relabeling the remaining indices so that they are the first $2(N-1)$ positive integers.

We let $X_{\text {simple }}\left(\Omega ; x_{1}, x_{2}\right)$ denote the set of continuous simple unparameterized curves in $\Omega$ connecting $x_{1}$ and $x_{2}$ such that they only touch the boundary $\partial \Omega$ in $\left\{x_{1}, x_{2}\right\}$. When $\kappa \in$ 
$(0,4]$, the chordal $\mathrm{SLE}_{\kappa}$ curve belongs to this space almost surely. Also, when $N \geq 2$, we let $X_{\text {simple }}^{\alpha}\left(\Omega ; x_{1}, \ldots, x_{2 N}\right)$ denote the set of families $\left(\eta_{1}, \ldots, \eta_{N}\right)$ of pairwise disjoint curves, where $\eta_{j} \in X_{\text {simple }}\left(\Omega ; x_{a_{j}}, x_{b_{j}}\right)$ for all $j \in\{1, \ldots, N\}$.

Definition 1.1. Let $\kappa \in(0,4]$. For $N \geq 2$ and for any link pattern $\alpha \in \mathrm{LP}_{N}$, we call a probability measure on the families $\left(\eta_{1}, \ldots, \eta_{N}\right) \in X_{\text {simple }}^{\alpha}\left(\Omega ; x_{1}, \ldots, x_{2 N}\right)$ a global $N$ $\mathrm{SLE}_{\kappa}$ associated to $\alpha$ if, for each $j \in\{1, \ldots, N\}$, the conditional law of the curve $\eta_{j}$ given $\left\{\eta_{1}, \ldots, \eta_{N}\right\} \backslash\left\{\eta_{j}\right\}$ is the chordal SLE $\kappa_{\kappa}$ connecting $x_{a_{j}}$ and $x_{b_{j}}$ in the connected component of the domain $\Omega \backslash \bigcup_{i \neq j} \eta_{i}$ containing the endpoints $x_{a_{j}}$ and $x_{b_{j}}$ of $\eta_{j}$ on its boundary.

THEOREM 1.2. Let $\kappa \in(0,4]$ and let $\left(\Omega ; x_{1}, \ldots, x_{2 N}\right)$ be a polygon with $N \geq 1$. For any $\alpha \in \mathrm{LP}_{N}$, there exists a unique global $N-\mathrm{SLE}_{\kappa}$ associated to $\alpha$.

The existence part of Theorem 1.2 is already known; see [21, 25, 31]. We briefly review the construction in Section 3.1. J. Miller and S. Sheffield proved the uniqueness part of Theorem 1.2 for $N=2$ in [28], Theorem 4.1, making use of a coupling of the SLE with the Gaussian free field. Unfortunately, this proof does not apply ${ }^{1}$ in general for $N \geq 3$ commuting SLEs. In the present article, we first give a different proof for the existence and uniqueness when $N=2$ by a Markov chain argument (in Section 3.2), and then generalize the uniqueness proof for all $N \geq 3$ (in Section 3.3). Our proof also gives exponential convergence rate for the Markov chain.

Lastly, let us note that Definition 1.1 implies the following cascade property. Suppose that the collection of random curves $\left(\eta_{1}, \ldots, \eta_{N}\right) \in X_{\text {simple }}^{\alpha}\left(\Omega ; x_{1}, \ldots, x_{2 N}\right)$ has the law of a global $N-\mathrm{SLE}_{\kappa}$ associated to the link pattern $\alpha \in \operatorname{LP}_{N}$. Assume also that $\{j, j+1\} \in \alpha$ for some $j \in\{1, \ldots, N\}$, and let $\eta_{1}$ be the curve connecting $x_{j}$ and $x_{j+1}$. Then the conditional law of the curves $\left(\eta_{2}, \ldots, \eta_{N}\right)$ given $\eta_{1}$ is the global $(N-1)-\operatorname{SLE}_{\kappa}$ associated to $\alpha /\{j, j+1\}$.

1.2. Multiple interfaces in the critical planar Ising model. Next, we consider critical Ising interfaces in the scaling limit. Assuming that $\Omega$ is bounded, we let discrete domains $\left(\Omega^{\delta} ; x_{1}^{\delta}, \ldots, x_{2 N}^{\delta}\right)$ on the square lattice approximate $\left(\Omega ; x_{1}, \ldots, x_{2 N}\right)$ as $\delta \rightarrow 0$ (we will provide the details of the approximation scheme in Section 4 ), and we consider the critical Ising model (which we also define in Section 4 ) on $\Omega^{\delta}$ with the following alternating boundary conditions:

$$
\begin{cases}\oplus \text { on }\left(x_{2 j-1}^{\delta} x_{2 j}^{\delta}\right), & \text { for } j \in\{1, \ldots, N\}, \\ \ominus \text { on }\left(x_{2 j}^{\delta} x_{2 j+1}^{\delta}\right), & \text { for } j \in\{0,1, \ldots, N\},\end{cases}
$$

where $\left(x_{i}^{\delta} x_{i+1}^{\delta}\right)$ stands for the counterclockwise boundary arc from $x_{i}^{\delta}$ to $x_{i+1}^{\delta}$, with the convention that $x_{2 N}^{\delta}=x_{0}^{\delta}$ and $x_{2 N+1}^{\delta}=x_{1}^{\delta}$. With the alternating boundary conditions (1.1), in the configurations of the Ising model, $N$ random interfaces $\left(\eta_{1}^{\delta}, \ldots, \eta_{N}^{\delta}\right)$ connect pairwise the $2 N$ boundary points $x_{1}^{\delta}, \ldots, x_{2 N}^{\delta}$, forming a planar connectivity encoded in a link pattern $\vartheta^{\delta} \in \mathrm{LP}_{N}$. See Figure 1 for an illustration.

To understand the scaling limit of the interfaces, we must specify the topology in which the convergence of the curves occurs. Thus, we let $X$ denote the set of planar oriented curves, that is, continuous mappings from $[0,1]$ to $\mathbb{C}$ modulo reparameterization. We equip $X$ with the metric

$$
\operatorname{dist}(\eta, \tilde{\eta}):=\inf _{\varphi, \tilde{\varphi}} \sup _{t \in[0,1]}|\eta(\varphi(t))-\tilde{\eta}(\tilde{\varphi}(t))|
$$

\footnotetext{
${ }^{1}$ Another proof (which might be generalizable for $N \geq 3$ ) for the case of two curves recently appeared in a new Appendix to [30]. However, this proof does not give exponential convergence rate of the Markov chain discussed in Remark 3.8.
} 
where the infimum is taken over all increasing homeomorphisms $\varphi, \tilde{\varphi}:[0,1] \rightarrow[0,1]$. Then the metric space $(X, d)$ is complete and separable. On the space $X_{\text {simple }}^{\alpha}\left(\Omega ; x_{1}, \ldots, x_{2 N}\right)$, we use the metric

$$
\operatorname{dist}\left(\left(\eta_{1}, \ldots, \eta_{N}\right),\left(\tilde{\eta}_{1}, \ldots, \tilde{\eta}_{N}\right)\right):=\max _{1 \leq j \leq N} \operatorname{dist}\left(\eta_{j}, \tilde{\eta}_{j}\right)
$$

Proposition 1.3. Let $\alpha \in \operatorname{LP}_{N}$. Then, as $\delta \rightarrow 0$, conditionally on the event $\left\{\vartheta^{\delta}=\alpha\right\}$, the law of the collection $\left(\eta_{1}^{\delta}, \ldots, \eta_{N}^{\delta}\right)$ of critical Ising interfaces converges weakly to the global $N-\mathrm{SLE}_{3}$ associated to $\alpha$. In particular, as $\delta \rightarrow 0$, the law of a single curve $\eta_{j}^{\delta}$ in this collection connecting two points $x_{a}$ and $x_{b}$ converges weakly to a conformal image of the Loewner chain with driving function given by equation (3.14) in Section 3.4 with $\kappa=3$.

We prove Proposition 1.3 in Section 4.4, where we also define the Ising model and discuss some of its main features. The key ingredients in the proof are results from [10,19] for the relative compactness of the curves, a technical RSW estimate [5] to rule out pathological behavior, and convergence of one interface [5] combined with Theorem 1.2 for the identification of the limit.

1.3. Multiple interfaces in the critical planar FK-Ising model. In Section 4, we also define and discuss the random-cluster models, whose interfaces conjecturally converge to $\mathrm{SLE}_{\kappa}$ curves with $\kappa \in(4,6]$. Using the discrete holomorphic observable, the convergence has been rigorously proven for the case of the FK-Ising model with $\kappa=16 / 3$ for a single interface [5] and two interfaces [20]—we provide with a proof for the general case. Hence, we consider the critical FK-Ising model on $\Omega^{\delta}$ with the following alternating boundary conditions (illustrated in Figure 5):

$$
\begin{cases}\text { wired on }\left(x_{2 j-1}^{\delta} x_{2 j}^{\delta}\right), & \text { for } j \in\{1, \ldots, N\}, \\ \text { free on }\left(x_{2 j}^{\delta} x_{2 j+1}^{\delta}\right), & \text { for } j \in\{0,1, \ldots, N\} .\end{cases}
$$

As in the case of the Ising model, $N$ interfaces $\left(\eta_{1}^{\delta}, \ldots, \eta_{N}^{\delta}\right)$ connect pairwise the $2 N$ boundary points $x_{1}^{\delta}, \ldots, x_{2 N}^{\delta}$, forming a planar connectivity encoded in a link pattern $\vartheta^{\delta} \in \operatorname{LP}_{N}$. However, this time the scaling limits are not simple curves, and we need to extend the definition of a global multiple $\mathrm{SLE}_{\kappa}$ to include the range $\kappa \in(4,6]$. For this, we let $X_{0}(\Omega ; x, y)$ denote the closure of the space $X_{\text {simple }}(\Omega ; x, y)$ in the metric topology of $(X$, dist). Note that the curves in $X_{0}(\Omega ; x, y)$ may have multiple points but no self-crossings. In particular, for all $\kappa>4$, the chordal $\mathrm{SLE}_{\kappa}$ curve belongs to this space almost surely.

Then, for each $N \geq 2$ and every $\alpha=\left\{\left\{a_{1}, b_{1}\right\}, \ldots,\left\{a_{N}, b_{N}\right\}\right\} \in \mathrm{LP}_{N}$, we denote by $X_{0}^{\alpha}\left(\Omega ; x_{1}, \ldots, x_{2 N}\right)$ the collection of curves $\left(\eta_{1}, \ldots, \eta_{N}\right)$ such that, for each $j \in\{1, \ldots, N\}$, we have $\eta_{j} \in X_{0}\left(\Omega ; x_{a_{j}}, x_{b_{j}}\right)$ and $\eta_{j}$ does not disconnect any two points $x_{a}, x_{b}$ such that $\{a, b\} \in \alpha$ from each other. Note that $X_{0}^{\alpha}\left(\Omega ; x_{1}, \ldots, x_{2 N}\right)$ is not complete. Above, the global $N-\mathrm{SLE}_{\kappa}$ was defined for $\kappa \in(0,4]$-we now extend this definition to all $\kappa \in(0,8)$ by replacing $X_{\text {simple }}^{\alpha}\left(\Omega ; x_{1}, \ldots, x_{2 N}\right)$ with $X_{0}^{\alpha}\left(\Omega ; x_{1}, \ldots, x_{2 N}\right)$ in Definition 1.1 . Note that this definition would actually still formally make sense in the range $\kappa \geq 8$, but since for such values of $\kappa$, the $\mathrm{SLE}_{\kappa}$ process is described by a Peano curve, uniqueness of a multiple SLE clearly fails, as one can specify the common boundaries of the different curves in an arbitrary way while preserving the conditional distributions of individual curves.

PROPOSITION 1.4. Theorem 1.2 also holds for $\kappa=16 / 3$, and for any $\alpha \in \mathrm{LP}_{N}$, as $\delta \rightarrow$ 0 , conditionally on the event $\left\{\vartheta^{\delta}=\alpha\right\}$, the law of the collection $\left(\eta_{1}^{\delta}, \ldots, \eta_{N}^{\delta}\right)$ of critical FKIsing interfaces converges weakly to the global $N-\mathrm{SLE}_{16 / 3}$ associated to $\alpha$. 
We prove Proposition 1.4 in Sections 4.2 and 4.3 (the proof is summarized in Section 4.2). The relative compactness of the curves is similar as in the Ising model. To show that the scaling limit is a global multiple $\mathrm{SLE}_{16 / 3}$, we use the convergence of one interface [5] combined with technical analysis using the RSW estimates [10]. To prove the uniqueness of the limit, we use a Markov chain argument similar to the proof of Theorem 1.2, thereby also establishing the uniqueness of the global multiple $\mathrm{SLE}_{\kappa}$ for $\kappa=16 / 3$. To this end, a priori estimates from the discrete model give us strong enough control of the curves (replacing the SLE analysis used for Theorem 1.2 in Section 3).

REMARK 1.5. Similar arguments as presented in Sections 4.2 and 4.3 combined with the results of $[4,38]$ show that there also exists a unique global multiple $\operatorname{SLE}_{\kappa}$ for $\kappa=6$ with any given connectivity pattern; and Proposition 1.4 holds for the critical site percolation on the triangular lattice with $\kappa=6$.

2. Preliminaries. In this section, we give some preliminary results, for use in subsequent sections. In Section 2.1, we discuss Brownian excursions and the Brownian loop measure. These concepts are needed frequently in Sections 2 and 3. In Sections 2.2 and 2.3, we define the chordal $\mathrm{SLE}_{\kappa}$ and study its relationships in different domains via so-called boundary perturbation properties. In Section 2.4, we give a crucial coupling result for SLEs in different domains. This coupling is needed in the proof of Theorem 1.2 in Section 3.

2.1. Brownian excursions and Brownian loop measure. We call a polygon $(\Omega ; x, y)$ with two marked points a Dobrushin domain. Given two boundary points $x, y \in \partial \Omega$, we denote by $(y x)$ the counterclockwise arc of $\partial \Omega$ from $y$ to $x$. Also, if $U \subset \Omega$ is a simply connected subdomain that agrees with $\Omega$ in neighborhoods of $x$ and $y$, we say that $U$ is a Dobrushin subdomain of $\Omega$. For a Dobrushin domain $(\Omega ; x, y)$, the Brownian excursion measure $v(\Omega ;(y x))$ is a conformally invariant measure on Brownian excursions in $\Omega$ with their two endpoints on the arc $(y x)$; see [27], Section 3, for definitions. It is a $\sigma$-finite infinite measure, with the following restriction property: for any Dobrushin subdomain $U \subset \Omega$ that agrees with $\Omega$ in a neighborhood of the arc $(y x)$, we have

$$
v(\Omega ;(y x))\left[\cdot \mathbb{1}_{\{e \subset U\}}\right]=v(U ;(y x))[\cdot] .
$$

For $\xi \geq 0$, we call a Poisson point process with intensity $\xi \nu(\Omega ;(y x))$ a Brownian excursion soup.

Whenever $x$ and $y$ lie on sufficiently regular boundary segments of $\Omega$, we define the boundary Poisson kernel $H_{\Omega}(x, y)$ as the unique function which in the upper-half plane $\mathbb{H}=\{z \in \mathbb{C}: \operatorname{Im}(z)>0\}$ is given by

$$
H_{\mathbb{H}}(x, y)=|y-x|^{-2}, \quad x, y \in \mathbb{R}
$$

and which in $\Omega$ is defined via conformal covariance: for any conformal map $\varphi: \Omega \rightarrow \varphi(\Omega)$, we have

$$
H_{\Omega}(x, y)=\left|\varphi^{\prime}(x) \varphi^{\prime}(y)\right| H_{\varphi(\Omega)}(\varphi(x), \varphi(y)),
$$

and in particular, $H_{\Omega}(x, y):=\left|\varphi^{\prime}(x) \varphi^{\prime}(y)\right| H_{\mathbb{H}}(\varphi(x), \varphi(y))$, with $\varphi: \Omega \rightarrow \mathbb{H}$.

LemMA 2.1. Let $(\Omega ; x, y)$ be a Dobrushin domain with $x, y$ on sufficiently regular boundary segments. Let $U, V \subset \Omega$ be two Dobrushin subdomains that agree with $\Omega$ in a neighborhood of the arc $(y x)$. Then we have

$$
\begin{aligned}
H_{\Omega}(x, y) & \geq H_{U}(x, y), \\
H_{\Omega}(x, y) H_{U \cap V}(x, y) & \geq H_{U}(x, y) H_{V}(x, y) .
\end{aligned}
$$


PROOF. The inequality (2.3) follows from (2.2). To prove (2.4), let $\mathcal{P}$ be a Brownian excursion soup with intensity $v(\Omega ;(y x))$. The union of excursions in $\mathcal{P}$ satisfies the so-called one-sided restriction property (see, e.g., [41], Theorem 8), which implies that $\mathbb{P}[e \subset U \forall e \in$ $\mathcal{P}]=\left|\varphi^{\prime}(x) \varphi^{\prime}(y)\right|$, where $\varphi$ is any conformal map from $U$ onto $\Omega$ fixing $x$ and $y$. Combining with (2.2), we obtain

$$
\mathbb{P}[e \subset U \forall e \in \mathcal{P}]=\frac{H_{U}(x, y)}{H_{\Omega}(x, y)} .
$$

Now, denote by $\mathcal{P}_{V}$ the collection of excursions in $\mathcal{P}$ that are contained in $V$. By (2.1), we know that $\mathcal{P}_{V}$ is a Brownian excursion soup with intensity $v(V ;(y x))$. The property (2.4) now follows from

$$
\frac{H_{U \cap V}(x, y)}{H_{V}(x, y)}=\mathbb{P}\left[e \subset U \forall e \in \mathcal{P}_{V}\right] \geq \mathbb{P}[e \subset U \forall e \in \mathcal{P}]=\frac{H_{U}(x, y)}{H_{\Omega}(x, y)} .
$$

This concludes the proof.

The Brownian loop measure $\mu(\Omega)$ is a conformally invariant measure on unrooted Brownian loops in $\Omega$; see, for example, [27], Sections 3 and 4 , for definitions. It is a $\sigma$-finite infinite measure, which has the following restriction property: for any subdomain $U \subset \Omega$, we have

$$
\mu(\Omega)\left[\cdot \mathbb{1}_{\{\ell \subset U\}}\right]=\mu(U)[\cdot] .
$$

For $\xi \geq 0$, we call a Poisson point process with intensity $\xi \mu(\Omega)$ a Brownian loop soup. This notion will be needed in Section 2.4 .

Given two disjoint subsets $V_{1}, V_{2} \subset \Omega$, we denote by $\mu\left(\Omega ; V_{1}, V_{2}\right)$ the Brownian loop measure of loops in $\Omega$ that intersect both $V_{1}$ and $V_{2}$. In other words,

$$
\mu\left(\Omega ; V_{1}, V_{2}\right):=\mu\left\{\ell: \ell \subset \Omega, \ell \cap V_{1} \neq \varnothing, \ell \cap V_{2} \neq \varnothing\right\} .
$$

If $V_{1}, V_{2}$ are at positive distance from each other, both of them are closed, and at least one of them is compact, then we have $0 \leq \mu\left(\Omega ; V_{1}, V_{2}\right)<\infty$. Furthermore, the measure $\mu\left(\Omega ; V_{1}, V_{2}\right)$ is conformally invariant: we have $\mu\left(\varphi(\Omega) ; \varphi\left(V_{1}\right), \varphi\left(V_{2}\right)\right)=\mu\left(\Omega ; V_{1}, V_{2}\right)$ for any conformal map $\varphi: \Omega \rightarrow f(\Omega)$.

For $n$ disjoint subsets $V_{1}, \ldots, V_{n}$ of $\Omega$, we denote by $\mu\left(\Omega ; V_{1}, \ldots, V_{n}\right)$ the Brownian loop measure of loops in $\Omega$ that intersect all of $V_{1}, \ldots, V_{n}$. Again, provided that $V_{j}$ are closed and at least one of them is compact, $\mu\left(\Omega ; V_{1}, \ldots, V_{n}\right)$ is finite. This quantity will be needed in Section 3.

2.2. Loewner chains and the Schramm-Loewner evolution. An $\mathbb{H}-$-hull is a compact subset $K$ of $\overline{\mathbb{H}}$ such that $\mathbb{H} \backslash K$ is simply connected. Riemann's mapping theorem implies that for any hull $K$, there exists a unique conformal map $g_{K}$ from $\mathbb{H} \backslash K$ onto $\mathbb{H}$ such that $\lim _{z \rightarrow \infty}\left|g_{K}(z)-z\right|=0$. Such a map $g_{K}$ is called the conformal map from $\mathbb{H} \backslash K$ onto $\mathbb{H}$ normalized at $\infty$. By standard estimates of conformal maps, the derivative of this map satisfies

$$
0<g_{K}^{\prime}(x) \leq 1 \quad \text { for all } x \in \mathbb{R} \backslash K .
$$

In fact, this derivative can be viewed as the probability that the Brownian excursion in $\mathbb{H}$ from $x$ to $\infty$ avoids the hull $K$; see [23].

Consider a family of conformal maps $\left(g_{t}, t \geq 0\right)$ which solve the Loewner equation: for each $z \in \mathbb{H}$,

$$
\partial_{t} g_{t}(z)=\frac{2}{g_{t}(z)-W_{t}} \quad \text { and } \quad g_{0}(z)=z
$$


where $\left(W_{t}, t \geq 0\right)$ is some real-valued continuous function, called the driving function. Also, denote $K_{t}:=\overline{\left\{z \in \mathbb{H}: T_{z} \leq t\right\}}$, where

$$
T_{z}:=\sup \left\{t \geq 0: \inf _{s \in[0, t]}\left|g_{s}(z)-W_{s}\right|>0\right\}
$$

is the swallowing time of the point $z$. Then $g_{t}$ is the unique conformal map from $H_{t}:=\mathbb{H} \backslash K_{t}$ onto $\mathbb{H}$ normalized at $\infty$. The collection of $\mathbb{H}$-hulls $\left(K_{t}, t \geq 0\right)$ associated with such maps is called a Loewner chain. We say that $\left(K_{t}, t \geq 0\right)$ is generated by the continuous curve $(\gamma(t), t \geq 0)$ if, for any $t \geq 0$, the unbounded connected component of $\mathbb{H} \backslash \gamma[0, t]$ coincides with $H_{t}=\mathbb{H} \backslash K_{t}$.

In this article, we are concerned with particular hulls generated by curves. For $\kappa \geq 0$, the random Loewner chain $\left(K_{t}, t \geq 0\right)$ driven by $W_{t}=\sqrt{\kappa} B_{t}$, where $\left(B_{t}, t \geq 0\right)$ is a standard Brownian motion, is called the (chordal) Schramm-Loewner Evolution, or $\mathrm{SLE}_{\kappa}$, in $\mathbb{H}$ from 0 to $\infty$. S. Rohde and O. Schramm proved in [32] that this Loewner chain is almost surely generated by a continuous transient curve $\gamma$, with $|\gamma(t)| \rightarrow \infty$ as $t \rightarrow \infty$, the SLE $_{\kappa}$ curve. This random curve exhibits the following phase transitions in the parameter $\kappa$ : when $\kappa \in$ $[0,4]$, it is a simple curve; whereas when $\kappa>4$, it has self-touchings, being space-filling if $\kappa \geq 8$. The law of the $\mathrm{SLE}_{\kappa}$ curve is a probability measure on the space $X_{0}(\mathbb{H} ; 0, \infty)$, and we denote it by $\mathbb{P}(\mathbb{H} ; 0, \infty)$.

By conformal invariance, we can define the $\operatorname{SLE}_{\kappa}$ probability measure $\mathbb{P}(\Omega ; x, y)$ in any simply connected domain $\Omega$ with two marked boundary points $x, y \in \partial \Omega$ (around which $\partial \Omega$ is locally connected) via pushforward of a conformal map: if $\gamma \sim \mathbb{P}(\mathbb{H} ; 0, \infty)$, then we have $\varphi(\gamma) \sim \mathbb{P}(\Omega ; x, y)$, where $\varphi: \mathbb{H} \rightarrow \Omega$ is any conformal map such that $\varphi(0)=x$ and $\varphi(\infty)=$ $y$. In fact, by the results of O. Schramm [34], the $\left(\mathrm{SLE}_{\kappa}\right)_{\kappa \geq 0}$ are the only probability measures on curves $\gamma \in X_{0}(\Omega ; x, y)$ satisfying conformal invariance and the following domain Markov property: given an initial segment $\gamma[0, \tau]$ of the $\operatorname{SLE}_{\kappa}$ curve $\gamma \sim \mathbb{P}(\Omega ; x, y)$ up to a stopping time $\tau$, the conditional law of the remaining piece $\gamma[\tau, \infty)$ is the law $\mathbb{P}\left(\Omega \backslash K_{\tau} ; \gamma(\tau), y\right)$ of the $\mathrm{SLE}_{\kappa}$ curve in the complement of the hull $K_{\tau}$ of the initial segment from the tip $\gamma(\tau)$ to $y$.

2.3. Boundary perturbation for SLEs. The chordal $\operatorname{SLE}_{\kappa}$ curve $\gamma \sim \mathbb{P}(\Omega ; x, y)$ has a natural boundary perturbation property, where its law in a Dobrushin subdomain of $\Omega$ is given by weighting by a factor involving the Brownian loop measure and the boundary Poisson kernel. More precisely, when $\kappa \in(0,4]$, the $\mathrm{SLE}_{\kappa}$ is a simple curve only touching the boundary at its endpoints, and its law in the subdomain is absolutely continuous with respect to its law in $\Omega$, as we state in the next Lemma 2.2. However, for $\kappa>4$, we cannot have such an absolute continuity property, because the $\mathrm{SLE}_{\kappa}$ has a positive chance to hit the boundary of $\Omega$. Nevertheless, in Lemma 2.3 we show that if we restrict the two processes in a smaller domain, then we retain the absolute continuity for $\kappa \in(4,8)$.

Throughout this article, we use the following real parameters, depending on $\kappa>0$ :

$$
h=\frac{6-\kappa}{2 \kappa} \quad \text { and } \quad c=\frac{(3 \kappa-8)(6-\kappa)}{2 \kappa} .
$$

Lemma 2.2. Let $\kappa \in(0,4]$. Let $(\Omega ; x, y)$ be a Dobrushin domain and $U \subset \Omega$ a Dobrushin subdomain. Then the $\mathrm{SLE}_{\kappa}$ in $U$ connecting $x$ and $y$ is absolutely continuous with respect to the $\mathrm{SLE}_{\kappa}$ in $\Omega$ connecting $x$ and $y$, with Radon-Nikodym derivative given by

$$
\frac{\mathrm{d} \mathbb{P}(U ; x, y)}{\mathrm{d} \mathbb{P}(\Omega ; x, y)}(\gamma)=\left(\frac{H_{\Omega}(x, y)}{H_{U}(x, y)}\right)^{h} \mathbb{1}_{\{\gamma \subset U\}} \exp (c \mu(\Omega ; \gamma, \Omega \backslash U)) \text {. }
$$


Proof. See [23], Section 5, and [21], Proposition 3.1.

The next lemma is a consequence of results in [23, 27]. We briefly summarize the proof.

Lemma 2.3. Let $\kappa \in(4,8)$. Let $(\Omega ; x, y)$ be a Dobrushin domain. Let $\Omega^{L} \subset U \subset \Omega$ be Dobrushin subdomains such that $\Omega^{L}$ and $\Omega$ agree in a neighborhood of the arc (yx) and $\operatorname{dist}\left(\Omega^{L}, \Omega \backslash U\right)>0$. Then we have

$$
\mathbb{1}_{\left\{\gamma \subset \Omega^{L}\right\}} \frac{\mathrm{d} \mathbb{P}(U ; x, y)}{\mathrm{d} \mathbb{P}(\Omega ; x, y)}(\gamma)=\left(\frac{H_{\Omega}(x, y)}{H_{U}(x, y)}\right)^{h} \mathbb{1}_{\left\{\gamma \subset \Omega^{L}\right\}} \exp (c \mu(\Omega ; \gamma, \Omega \backslash U)) .
$$

PROOF. By conformal invariance, we may assume that the domain under consideration is $(\Omega ; x, y)=(\mathbb{H} ; 0, \infty)$. Let $\gamma \sim \mathbb{P}(\mathbb{H} ; 0, \infty)$, let $\left(W_{t}, t \geq 0\right)$ be its driving function, and $\left(g_{t}, t \geq 0\right)$ the corresponding conformal maps. Let $\varphi$ be the conformal map from $U$ onto $\mathbb{H}$ normalized at $\infty$. On the event $\left\{\gamma \subset \Omega^{L}\right\}$, define $T$ to be the first time when $\gamma$ disconnects $\mathbb{H} \backslash U$ from $\infty$.

Denote by $K_{t}$ the hull of $\gamma[0, t]$. For $t<T$, let $\tilde{g}_{t}$ be the conformal map from $\mathbb{H} \backslash \varphi\left(K_{t}\right)$ onto $\mathbb{H}$, and let $\varphi_{t}$ be the conformal map from $g_{t}\left(U \backslash K_{t}\right)$ onto $\mathbb{H}$, both normalized at $\infty$. Then we have $\tilde{g}_{t} \circ \varphi=\varphi_{t} \circ g_{t}$. Now we define, for $t<T$,

$$
M_{t}:=\varphi_{t}^{\prime}\left(W_{t}\right)^{h} \exp \left(-c \int_{0}^{t} \frac{S \varphi_{s}\left(W_{s}\right)}{6} \mathrm{~d} s\right),
$$

where $S f$ is the Schwarzian derivative. ${ }^{2}$ It was proved in [23], Proposition 5.3, that $M_{t}$ is a local martingale. Furthermore, using Itô's formula, one can show that the law of $\gamma$ weighted by $M_{t}$ is $\mathbb{P}(U ; 0, \infty)$ up to time $t$. Also, it follows from [24], Proposition 5.22, (see also [27], Section 7) that

$$
-\int_{0}^{t} \frac{S \varphi_{s}\left(W_{s}\right)}{6} \mathrm{~d} s=\mu(\mathbb{H} ; \gamma[0, t], \mathbb{H} \backslash U) .
$$

Now, on the event $\left\{\gamma \subset \Omega^{L}\right\}$, there exists a constant $\varepsilon=\varepsilon\left(\mathbb{H}, \Omega^{L}, U\right)>0$ such that for $t<T$, we have $\varepsilon \leq \varphi_{t}^{\prime}\left(W_{t}\right) \leq 1$. When $\kappa \in(4,6]$, we have $h \geq 0$ and $c \geq 0$, and thus, on the event $\left\{\gamma \subset \Omega^{L}\right\}$, we have $M_{t} \leq \exp \left(c \mu\left(\mathbb{H} ; \Omega^{L}, \mathbb{H} \backslash U\right)\right)$. When $\kappa \in(6,8)$, we have $h \leq 0$ and $c \leq 0$, and in this case, we have $M_{t} \leq \varepsilon^{h}$. In conclusion, in either case, $\left(M_{t}, t<T\right)$ is uniformly bounded on the event $\left\{\gamma \subset \Omega^{L}\right\}$, and as $t \rightarrow T$, we have $\varphi_{t}^{\prime}\left(W_{t}\right) \rightarrow 1$ almost surely, so

$$
M_{t} \rightarrow M_{T}:=\exp (c \mu(\mathbb{H} ; \gamma[0, T], \mathbb{H} \backslash U)), \quad \text { as } t \rightarrow T .
$$

The assertion now follows by taking into account that $M_{0}=\varphi^{\prime}(0)^{h}$ and recalling the identity (2.2).

2.4. A crucial coupling result for SLEs. We finish this preliminary section with a result from [42], which says that we can construct SLEs from the Brownian loop soup and the Brownian excursion soup. This gives a coupling of SLEs in two Dobrushin domains $U \subset \Omega$, which will be crucial in our proof of Theorem 1.2 (for Lemma 3.5 for $\kappa \in[8 / 3,4]$ ).

Let $(\Omega ; x, y)$ be a Dobrushin domain. Let $\mathcal{L}$ be a Brownian loop soup with intensity $c \mu(\Omega)$, and $\mathcal{P}$ a Brownian excursion soup with intensity $h v(\Omega ;(y x))$, where $c=c(\kappa)$ and $h=h(\kappa)$ are defined in (2.5) and $\kappa \in[8 / 3,4]$. (Note that for $\kappa \in[8 / 3,4]$, we have $c \in[0,1]$ and $h \in[1 / 4,5 / 8]$.) 

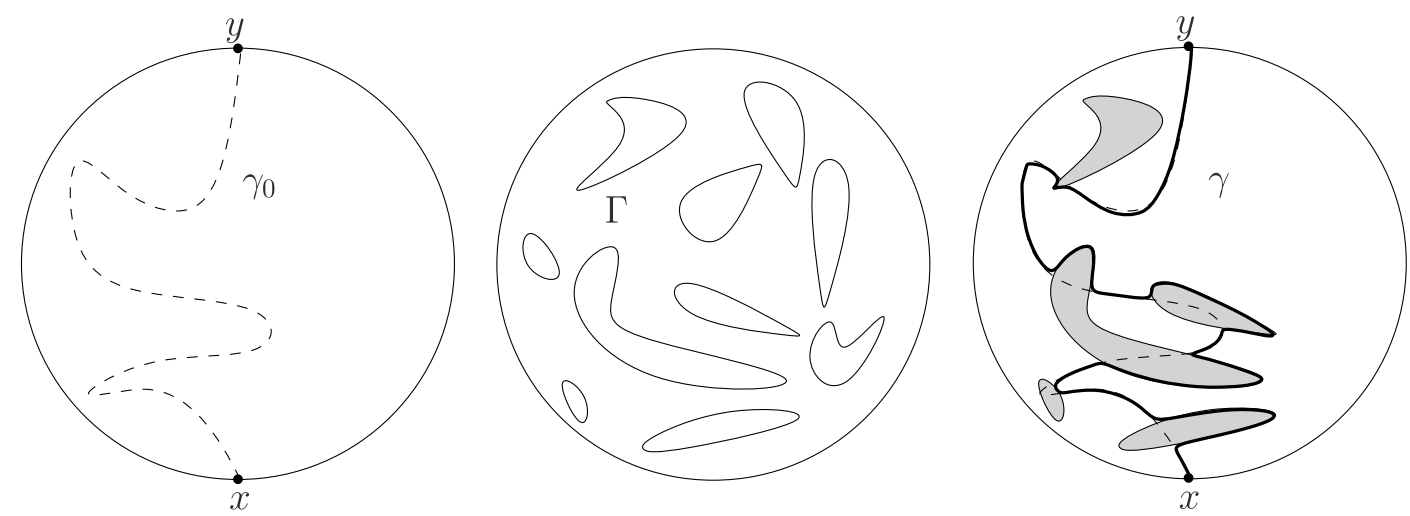

FIG. 2. In the left panel, $\gamma_{0}$ is the right boundary of all Brownian excursions in $\mathcal{P}$. In the middle panel, $\Gamma$ is the family of all outer boundaries of the outermost elements of the clusters of Brownian loops in $\mathcal{L}$. In the right panel, $\gamma$ is the right boundary of the union of $\gamma_{0}$ and all loops in $\Gamma$ that intersect $\gamma_{0}$. By [42], Theorem 1.1, we find that $\gamma \sim \mathbb{P}(\Omega ; x, y)$.

We say that two loops $\ell$ and $\ell^{\prime}$ in $\mathcal{L}$ belong to the same cluster if there exists a finite chain of loops $\ell_{0}, \ldots, \ell_{n}$ in $\mathcal{L}$ such that $\ell_{0}=\ell, \ell_{n}=\ell^{\prime}$, and $\ell_{j} \cap \ell_{j-1} \neq \varnothing$ for all $j \in\{1, \ldots, n\}$. We denote by $\overline{\mathcal{C}}$ the family of all closures of the loop-clusters and by $\Gamma$ the family of all outer boundaries of the outermost elements of $\overline{\mathcal{C}}$. Then $\Gamma$ forms a collection of disjoint simple loops, called the $\mathrm{CLE}_{\kappa}$ for $\kappa \in(8 / 3,4]$; see [37].

Finally, we define $\gamma_{0}$ to be the right boundary of the union of all excursions $e \in \mathcal{P}$ and $\gamma$ the boundary of the union of $\gamma_{0}$ and all loops in $\Gamma$ that it intersects, as illustrated in Figure 2.

LemMA 2.4. Let $\kappa \in[8 / 3,4]$. Let $(\Omega ; x, y)$ be a Dobrushin domain and define $\mathcal{L}, \mathcal{P}, \Gamma$, $\gamma_{0}$, and $\gamma$ as above. Then $\gamma$ has the law of the $\mathrm{SLE}_{\kappa}$ in $\Omega$ connecting $x$ and $y$.

PROOF. When $\kappa=8 / 3$, the curve $\gamma$ is the same as $\gamma_{0}$, and it satisfies the so-called onesided restriction property, which uniquely identifies its law with the $\mathrm{SLE}_{8 / 3}$ by [23], Theorem 8.4, and [41], Theorem 8. For $\kappa \in(8 / 3,4]$, the assertion was proved in [42], Theorem 1.1.

From Lemma 2.4, we see that $\mathrm{SLE}_{\kappa}$ curves in different domains can be coupled in the following way. Let $U \subset \Omega$ by a Dobrushin subdomain that agrees with $\Omega$ in a neighborhood of the arc $(y x)$. Take $\mathcal{L}, \mathcal{P}, \Gamma, \gamma_{0}$ and $\gamma$ as in the above lemma. Let $\mathcal{P}_{U}$ and $\mathcal{L}_{U}$, respectively, be the collections of excursions in $\mathcal{P}$ and loops in $\mathcal{L}$ that are contained in $U$. Define $\eta_{0}$ to be the right boundary of the union of all excursions $e \in \mathcal{P}_{U}$, define $\Gamma_{U}$ to be the collection of all outer boundaries of the outermost clusters of $\mathcal{L}_{U}$ and $\eta$ to be the right boundary of the union of $\eta_{0}$ and all loops in $\Gamma_{U}$ that it intersects.

Corollary 2.5. Let $\kappa \in[8 / 3,4]$. Let $(\Omega ; x, y)$ be a Dobrushin domain and $U \subset \Omega a$ Dobrushin subdomain that agrees with $\Omega$ in a neighborhood of the arc $(y x)$. There exists a coupling $(\gamma, \eta)$ of $\gamma \sim \mathbb{P}(\Omega ; x, y)$ and $\eta \sim \mathbb{P}(U ; x, y)$ such that, almost surely, $\eta$ stays to the left of $\gamma$ and

$$
\mathbb{P}[\eta=\gamma]=\mathbb{P}[\gamma \subset U]
$$

PROOF. Lemma 2.4 and the above paragraph give the sought coupling.

REMARK 2.6. The coupling $(\gamma, \eta)$ of Corollary 2.5 is the one which maximizes the probability $\mathbb{P}[\eta=\gamma]$. 
3. Global multiple SLEs. This section concerns the existence and uniqueness of global multiple $\mathrm{SLE}_{\kappa}$ measures for $\kappa \in(0,4]$. Such global $N$-SLEs associated to all link patterns $\alpha \in \mathrm{LP}_{N}$ and all $\kappa \in(0,4]$ have been constructed in [21, 25, 31]. In Section 3.1, we briefly recall this construction, which immediately gives the existence part of Theorem 1.2. We prove the uniqueness part of Theorem 1.2 in Sections 3.2 and 3.3.

3.1. Construction of global multiple SLEs for $\kappa \leq 4$. Fix a polygon $\left(\Omega ; x_{1}, \ldots, x_{2 N}\right)$. For a link pattern $\alpha=\left\{\left\{a_{1}, b_{1}\right\}, \ldots,\left\{a_{N}, b_{N}\right\}\right\} \in \mathrm{LP}_{N}$, we let $\mathbb{P}_{\alpha}$ denote the product measure of $N$ independent chordal $\mathrm{SLE}_{\kappa}$ curves,

$$
\mathbb{P}_{\alpha}:=\bigotimes_{j=1}^{N} \mathbb{P}\left(\Omega ; x_{a_{j}}, x_{b_{j}}\right),
$$

and $\mathbb{E}_{\alpha}$ the expectation with respect to $\mathbb{P}_{\alpha}$. A global $N$-SLE $\mathrm{S}_{\kappa}$ associated to $\alpha$ can be constructed as the probability measure $\mathbb{Q}_{\alpha}^{\#}=\mathbb{Q}_{\alpha}^{\#}\left(\Omega ; x_{1}, \ldots, x_{2 N}\right)$ which is absolutely continuous with respect to $\mathbb{P}_{\alpha}$ with explicit Radon-Nikodym derivative given in (3.1) below. This formula involves a combinatorial expression $m_{\alpha}$ of Brownian loop measures, obtained by an inclusion-exclusion procedure that depends on $\alpha$. More precisely, for a configuration $\left(\eta_{1}, \ldots, \eta_{N}\right) \in X_{0}^{\alpha}\left(\Omega ; x_{1}, \ldots, x_{2 N}\right)$, we define

$$
m_{\alpha}\left(\Omega ; \eta_{1}, \ldots, \eta_{N}\right):=\sum_{\text {c.c. } \mathcal{C} \text { of } \Omega \backslash\left\{\eta_{1}, \ldots, \eta_{N}\right\}} m(\mathcal{C}),
$$

where the sum is over all the connected components (c.c.) of the complement of the curves, and

$$
\begin{aligned}
m(\mathcal{C}):= & \sum_{\substack{i_{1}, i_{2} \in \mathcal{B}(\mathcal{C}), i_{1} \neq i_{2}}} \mu\left(\Omega ; \eta_{i_{1}}, \eta_{i_{2}}\right)-\sum_{\substack{i_{1}, i_{2}, i_{3} \in \mathcal{B}(\mathcal{C}), i_{1} \neq i_{2} \neq i_{3} \neq i_{1}}} \mu\left(\Omega ; \eta_{i_{1}}, \eta_{i_{2}}, \eta_{i_{3}}\right) \\
& +\cdots+(-1)^{p} \mu\left(\Omega ; \eta_{j_{1}}, \ldots, \eta_{j_{p}}\right)
\end{aligned}
$$

is a combinatorial expression associated to the c.c. $\mathcal{C}$, where

$$
\mathcal{B}(\mathcal{C}):=\left\{j \in\{1, \ldots, N\}: \eta_{j} \subset \partial \mathcal{C}\right\}=\left\{j_{1}, \ldots, j_{p}\right\}
$$

denotes the set of indices $j$ for which the curve $\eta_{j}$ is a part of the boundary of $\mathcal{C}$. Now, we define the probability measure $\mathbb{Q}_{\alpha}^{\#}$ via

$$
\frac{\mathrm{d} \mathbb{Q}_{\alpha}^{\#}}{\mathrm{~d} \mathbb{P}_{\alpha}}\left(\eta_{1}, \ldots, \eta_{N}\right)=\frac{R_{\alpha}\left(\Omega ; \eta_{1}, \ldots, \eta_{N}\right)}{\mathbb{E}_{\alpha}\left[R_{\alpha}\left(\Omega ; \eta_{1}, \ldots, \eta_{N}\right)\right]},
$$

where $R_{\alpha}\left(\Omega ; \eta_{1}, \ldots, \eta_{N}\right):=\mathbb{1}_{\left\{\eta_{j} \cap \eta_{k}=\varnothing \forall j \neq k\right\}} \exp \left(c m_{\alpha}\left(\Omega ; \eta_{1}, \ldots, \eta_{N}\right)\right)$.

By [31], Proposition 3.3, this measure satisfies the defining property of a global multiple $\mathrm{SLE}_{\kappa}$, stated in Definition 1.1. Also, as observed in [31], equation (3.6), the expectation of $R_{\alpha}$ defines a conformally invariant and bounded function of the marked boundary points:

$$
0<f_{\alpha}\left(\Omega ; x_{1}, \ldots, x_{2 N}\right):=\mathbb{E}_{\alpha}\left[R_{\alpha}\left(\Omega ; \eta_{1}, \ldots, \eta_{N}\right)\right] \leq 1 .
$$

If $\left(\Omega ; x_{1}, \ldots, x_{2 N}\right)$ is a polygon and $U \subset \Omega$ a simply connected subdomain that agrees with $\Omega$ in neighborhoods of $x_{1}, \ldots, x_{2 N}$, we say that $U$ is a subpolygon of $\Omega$. When the marked points $x_{1}, \ldots, x_{2 N}$ lie on sufficiently regular boundary segments of $\Omega$, we may define, for all $\alpha \in \mathrm{LP}_{N}$, the functions

$$
\mathcal{Z}_{\alpha}\left(\Omega ; x_{1}, \ldots, x_{2 N}\right):=f_{\alpha}\left(\Omega ; x_{1}, \ldots, x_{2 N}\right) \prod_{\{a, b\} \in \alpha} H_{\Omega}\left(x_{a}, x_{b}\right)^{h},
$$


where $H_{\Omega}$ is the boundary Poisson kernel introduced in Section 2.1. Since $0<f_{\alpha} \leq 1$, we see that

$$
0<\mathcal{Z}_{\alpha}\left(\Omega ; x_{1}, \ldots, x_{2 N}\right) \leq \prod_{\{a, b\} \in \alpha} H_{\Omega}\left(x_{a}, x_{b}\right)^{h} .
$$

The functions $\mathcal{Z}_{\alpha}$ are called pure partition functions of multiple SLEs. Explicit formulas for them have been obtained when $\kappa=2$ [18], Theorem 4.1, and $\kappa=4$ [31], Theorem 1.5. For other values of $\kappa \in(0,8)$, formulas in (complicated) integral form have been found in [11, 22].

The multiple SLE probability measure $\mathbb{Q}_{\alpha}^{\#}$ has a useful boundary perturbation property. It serves as an analogue of Lemma 2.2 in our proof of Theorem 1.2.

Proposition 3.1 ([31], Proposition 3.4). Fix $\kappa \in(0,4]$, and let $\left(\Omega ; x_{1}, \ldots, x_{2 N}\right)$ be a polygon and $U \subset \Omega$ a subpolygon. Then, the probability measure $\mathbb{Q}_{\alpha}^{\#}\left(U ; x_{1}, \ldots, x_{2 N}\right)$ is absolutely continuous with respect to $\mathbb{Q}_{\alpha}^{\#}\left(\Omega ; x_{1}, \ldots, x_{2 N}\right)$, with Radon-Nikodym derivative

$$
\begin{aligned}
& \frac{\mathrm{d} \mathbb{Q}_{\alpha}^{\#}\left(U ; x_{1}, \ldots, x_{2 N}\right)}{\mathrm{d} \mathbb{Q}_{\alpha}^{\#}\left(\Omega ; x_{1}, \ldots, x_{2 N}\right)}\left(\eta_{1}, \ldots, \eta_{N}\right) \\
& \quad=\frac{\mathcal{Z}_{\alpha}\left(\Omega ; x_{1}, \ldots, x_{2 N}\right)}{\mathcal{Z}_{\alpha}\left(U ; x_{1}, \ldots, x_{2 N}\right)} \mathbb{1}_{\left\{\eta_{j} \subset U \forall j\right\}} \exp \left(c \mu\left(\Omega ; \Omega \backslash U, \bigcup_{j=1}^{N} \eta_{j}\right)\right) .
\end{aligned}
$$

Moreover, if $\kappa \leq 8 / 3$ and $x_{1}, \ldots, x_{2 N}$ lie on sufficiently regular boundary segments of $\Omega$, then we have

$$
\mathcal{Z}_{\alpha}\left(\Omega ; x_{1}, \ldots, x_{2 N}\right) \geq \mathcal{Z}_{\alpha}\left(U ; x_{1}, \ldots, x_{2 N}\right)
$$

3.2. Uniqueness for a pair of commuting SLEs. Next, we prove that the global 2-SLE ${ }_{\kappa}$ measures are unique. This result was proved by J. Miller and S. Sheffield [28], Theorem 4.1, using a coupling of the SLEs with the Gaussian free field (GFF). We present another proof not using this coupling. Our proof also generalizes to the case of $N \geq 3$ commuting SLE curves, whereas couplings with the GFF seem not to be useful in that case.

In this section, we focus on polygons with $N=2$. We call such a polygon $\left(\Omega ; x_{1}, x_{2}, x_{3}\right.$, $\left.x_{4}\right)$ a quad. Because the two connectivities $\alpha \in \mathrm{LP}_{2}$ of the curves are obtained from each other by a cyclic change of labeling of the marked boundary points, we may without loss of generality consider global 2-SLEs associated to $\alpha=\{\{1,4\},\{2,3\}\}$. Hence, throughout this section we consider pairs $\left(\eta^{L}, \eta^{R}\right)$ of simple curves such that $\eta^{L} \in X_{0}\left(\Omega ; x^{L}, y^{L}\right)$ and $\eta^{R} \in X_{0}\left(\Omega ; x^{R}, y^{R}\right)$, with $\eta^{L} \cap \eta^{R}=\varnothing$. We denote the space of such pairs by $X_{0}\left(\Omega ; x^{L}, x^{R}, y^{R}, y^{L}\right)$. Now, a probability measure on these pairs $\left(\eta^{L}, \eta^{R}\right)$ of curves is a global 2-SLE $\mathrm{SL}_{\kappa}$ if the conditional law of $\eta^{L}$ given $\eta^{R}$ is that of the $\operatorname{SLE}_{\kappa}$ connecting $x^{L}$ and $y^{L}$ in the connected component of $\Omega \backslash \eta^{R}$ containing $x^{L}$ and $y^{L}$ on its boundary, and vice versa.

PROPOSITION 3.2. For any $\kappa \in(0,4]$, there exists a unique global $2-\mathrm{SLE}_{\kappa}$ on the space $X_{0}\left(\Omega ; x^{L}, x^{R}, y^{R}, y^{L}\right)$.

COROLlary 3.3. Let $\kappa \in(0,4]$. For any $\alpha \in \mathrm{LP}_{2}$, there exists a unique global 2-SLE $\mathrm{S}_{\kappa}$ associated to $\alpha$.

PROOF. The two connectivities $\alpha \in \mathrm{LP}_{2}$ of the curves are obtained from each other by a cyclic change of labeling of the marked boundary points $x_{1}, x_{2}, x_{3}, x_{4}$. Thus, the assertion follows from Proposition 3.2. 
We prove Proposition 3.2 in the end of this section, after some technical lemmas. The idea is to show that the global 2-SLE ${ }_{\kappa}$ is the unique stationary measure of a Markov chain, which at each discrete time resamples one of the two curves according to its conditional law given the other one. In fact, the existence part is already well known (see, e.g., [21] and Section 3.1 of the present article), so we only need to prove the uniqueness. Nevertheless, as pointed out by the referee, our Markov chain coupling argument actually gives both the uniqueness and existence of the stationary measure, thanks to the following special case of the Doeblin condition.

LEMMA 3.4. Let $P$ be a Markov kernel on a measurable space $E$ satisfying uniform coupling in the sense that there exists $\theta \in(0,1)$ such that the total variation distance between images is uniformly bounded as

$$
\sup _{x, y \in E}\left\|\delta_{x} P-\delta_{y} P\right\|_{\mathrm{TV}} \leq \theta .
$$

Then there exists a unique $P$-stationary probability measure $\mathbb{P}$, and for every $x \in E$, the Markov chain of kernel $P$ starting at $x$ converges in distribution to $\mathbb{P}$.

PROOF. The key consequence of the uniform coupling (3.5) is that, whenever $\mathbb{P}_{1}$ and $\mathbb{P}_{2}$ are two probability measures on $E$, we have the upper bound $\left\|\mathbb{P}_{1} P-\mathbb{P}_{2} P\right\|_{\mathrm{TV}} \leq \theta \| \mathbb{P}_{1}-$ $\mathbb{P}_{2} \|_{\mathrm{TV}}$. Applying this to two stationary measures $\mathbb{P}_{1}$ and $\mathbb{P}_{2}$ readily implies the uniqueness. Now, let $\left\{X_{n}\right\}$ be a Markov chain of kernel $P$ starting from $x \in E$, and denote by $\mathbb{P}_{n}$ the law of $X_{n}$, that is, $\mathbb{P}_{n}=\delta_{x} P^{n}$. Then, for all $0 \leq n \leq m$, we have

$$
\left\|\mathbb{P}_{n}-\mathbb{P}_{m}\right\|_{\mathrm{TV}}=\left\|\delta_{x} P^{n}-\mathbb{P}_{m-n} P^{n}\right\|_{\mathrm{TV}} \leq \theta^{n}\left\|\delta_{x}-\mathbb{P}_{m-n}\right\|_{\mathrm{TV}} \leq \theta^{n}
$$

so the sequence $\left\{\mathbb{P}_{n}\right\}$ is Cauchy for the total variation distance. Thus, by the completeness of the space of measures, it converges to a limit $\mathbb{P}$ which is $P$-stationary, thus showing the existence.

The next key Lemmas 3.5 and 3.6 are needed in order to establish the uniform coupling for Lemma 3.4. The first one, Lemma 3.5, is crucial: the chordal $\mathrm{SLE}_{\kappa}$ in $\Omega$ always has a uniformly positive probability of staying in a subdomain of $\Omega$ in the following sense.

LemMA 3.5. Let $\kappa \in(0,4]$. Let $(\Omega ; x, y)$ be a Dobrushin domain. Let $\Omega^{L}, U \subset \Omega$ be Dobrushin subdomains such that $\Omega^{L}, U$, and $\Omega$ agree in a neighborhood of the arc $(y x)$. Suppose $\eta \sim \mathbb{P}(U ; x, y)$. Then there exists a constant $\theta=\theta\left(\Omega, \Omega^{L}\right)>0$ independent of $U$ such that $\mathbb{P}\left[\eta \subset \Omega^{L}\right] \geq \theta$.

PROOF. We prove the lemma separately for $\kappa \in[8 / 3,4]$ and $\kappa \in(0,8 / 3]$. For the former case, we make use of the coupling from Section 2.4. For the latter, technically easier case, we use properties of the Brownian loop measure from Section 2.1 and the SLE boundary perturbation property from Section 2.3.

When $\kappa \in[8 / 3,4]$, we have $c \geq 0$ by (2.5). Suppose $\gamma \sim \mathbb{P}(\Omega ; x, y)$ and denote by $D_{\eta}$ (resp. $D_{\gamma}$ ) the connected component of $U \backslash \eta$ (resp. $\Omega \backslash \gamma$ ) with $(y x)$ on its boundary. By Corollary 2.5, there exists a coupling of $\eta$ and $\gamma$ such that $D_{\eta} \subset D_{\gamma}$. Therefore, we have $\mathbb{P}\left[\eta \subset \Omega^{L}\right] \geq \mathbb{P}\left[\gamma \subset \Omega^{L}\right]>0$. This gives the assertion for $\kappa \in[8 / 3,4]$ with $\theta\left(\Omega, \Omega^{L}\right)=$ $\mathbb{P}\left[\gamma \subset \Omega^{L}\right]>0$.

When $\kappa \in(0,8 / 3]$, we have $c \leq 0$ by (2.5). Lemma 2.2 gives

$$
\mathbb{P}\left[\eta \subset \Omega^{L}\right]=\left(\frac{H_{\Omega}(x, y)}{H_{U}(x, y)}\right)^{h} \mathbb{E}\left[\mathbb{1}_{\left\{\gamma \subset \Omega^{L} \cap U\right\}} \exp (c \mu(\Omega ; \gamma, \Omega \backslash U))\right] .
$$


Note that, on the event $\left\{\gamma \subset \Omega^{L} \cap U\right\}$, we have

$$
\begin{aligned}
& \mu\left(\Omega ; \gamma, \Omega \backslash\left(\Omega^{L} \cap U\right)\right) \\
& \quad=\mu(\Omega ; \gamma, \Omega \backslash U)+\mu\left(\Omega ; \gamma, \Omega \backslash \Omega^{L}\right)-\mu\left(\Omega ; \gamma, \Omega \backslash \Omega^{L}, \Omega \backslash U\right) \\
& \quad=\mu(\Omega ; \gamma, \Omega \backslash U)+\mu\left(U ; \gamma, U \backslash \Omega^{L}\right) .
\end{aligned}
$$

Combining (3.6) and (3.7) and using Lemmas 2.1 and 2.2, we obtain

$$
\begin{aligned}
\mathbb{P}\left[\eta \subset \Omega^{L}\right] & =\left(\frac{H_{\Omega}(x, y)}{H_{U}(x, y)}\right)^{h} \mathbb{E}\left[\mathbb{1}_{\left\{\gamma \subset \Omega^{L} \cap U\right\}} \exp (c \mu(\Omega ; \gamma, \Omega \backslash U))\right] \\
& \geq\left(\frac{H_{\Omega}(x, y)}{H_{U}(x, y)}\right)^{h} \mathbb{E}\left[\mathbb{1}_{\left\{\gamma \subset \Omega^{L} \cap U\right\}} \exp \left(c \mu\left(\Omega ; \gamma, \Omega \backslash\left(\Omega^{L} \cap U\right)\right)\right)\right] \\
& =\left(\frac{H_{\Omega^{L} \cap U}(x, y)}{H_{U}(x, y)}\right)^{h} \\
& \geq\left(\frac{H_{\Omega^{L}}(x, y)}{H_{\Omega}(x, y)}\right)^{h} .
\end{aligned}
$$

This gives the assertion for $\kappa \in(0,8 / 3]$ with the lower bound

$$
\theta\left(\Omega, \Omega^{L}\right)=\left(\frac{H_{\Omega^{L}}(x, y)}{H_{\Omega}(x, y)}\right)^{h}>0 .
$$

Next, we prove that one can couple two SLEs in two Dobrushin subdomains of $\Omega$ in such a way that their realizations agree with a uniformly positive probability.

LEMMA 3.6. Let $\kappa \in(0,8)$. Let $(\Omega ; x, y)$ be a Dobrushin domain. Let $\Omega^{L} \subset V \subset U$, $\tilde{U} \subset \Omega$ be Dobrushin subdomains such that $\Omega^{L}$ and $\Omega$ agree in a neighborhood of the arc (yx) and $\operatorname{dist}\left(\Omega^{L}, \Omega \backslash V\right)>0$. Suppose $\eta \sim \mathbb{P}(U ; x, y)$ and $\tilde{\eta} \sim \mathbb{P}(\tilde{U} ; x, y)$. Then there exists a coupling $(\eta, \tilde{\eta})$ such that $\mathbb{P}\left[\eta=\tilde{\eta} \subset \Omega^{L}\right] \geq \theta$, where the constant $\theta=\theta\left(\Omega, \Omega^{L}, V\right)>0$ is independent of $U$ and $\tilde{U}$.

ProOF. First, we show that there exists a constant $p_{0}=p_{0}\left(\Omega, \Omega^{L}, V\right)>0$, independent of $U$ and $\tilde{U}$, such that $\mathbb{P}\left[\eta \subset \Omega^{L}\right] \geq p_{0}$. This is true for $\kappa \leq 4$ by Lemma 3.5 , so it remains to treat the case $\kappa \in(4,8)$. For this, we use the SLE boundary perturbation property from Section 2.3.

Let $\gamma \sim \mathbb{P}(\Omega ; x, y)$. By Lemma 2.3, we have

$$
\mathbb{P}\left[\eta \subset \Omega^{L}\right]=\left(\frac{H_{\Omega}(x, y)}{H_{U}(x, y)}\right)^{h} \mathbb{E}\left[\mathbb{1}_{\left\{\gamma \subset \Omega^{L}\right\}} \exp (c \mu(\Omega ; \gamma, \Omega \backslash U))\right] .
$$

When $\kappa \in(4,6]$, we have $c \geq 0$ and $h \geq 0$ by (2.5). Combining this with the inequality (2.3), we obtain

$$
\mathbb{P}\left[\eta \subset \Omega^{L}\right] \geq \mathbb{P}\left[\gamma \subset \Omega^{L}\right]
$$

On the other hand, when $\kappa \in(6,8)$, then (2.5) implies that $c \leq 0$ and $h \leq 0$. On the event $\left\{\gamma \subset \Omega^{L}\right\}$, we have $\mu(\Omega ; \gamma, \Omega \backslash U) \leq \mu\left(\Omega ; \Omega^{L}, \Omega \backslash V\right)$, so combining with (2.3), we obtain

$$
\mathbb{P}\left[\eta \subset \Omega^{L}\right] \geq\left(\frac{H_{\Omega}(x, y)}{H_{V}(x, y)}\right)^{h} \exp \left(c \mu\left(\Omega ; \Omega^{L}, \Omega \backslash V\right)\right) \mathbb{P}\left[\gamma \subset \Omega^{L}\right] .
$$

In either case, we have $\mathbb{P}\left[\eta \subset \Omega^{L}\right] \geq p_{0}$ with $p_{0}=p_{0}\left(\Omega, \Omega^{L}, V\right)>0$, independently of $U$ and $\tilde{U}$, as claimed. 
Next, we consider the relation between the two $\operatorname{SLE}_{\kappa}$ curves $\tilde{\eta}$ and $\eta$. Using Lemmas 2.2 and 2.3, we see that the law of $\tilde{\eta}$ restricted to $\left\{\tilde{\eta} \subset \Omega^{L}\right\}$ is absolutely continuous with respect to the law of $\eta$ restricted to $\left\{\eta \subset \Omega^{L}\right\}$, and the Radon-Nikodym derivative is given by

$$
R(\eta):=\left(\frac{H_{U}(x, y)}{H_{\tilde{U}}(x, y)}\right)^{h} \mathbb{1}_{\left\{\eta \subset \Omega^{L}\right\}} \exp (c \mu(U ; \eta, U \backslash \tilde{U})-c \mu(\tilde{U} ; \eta, \tilde{U} \backslash U)) .
$$

Now, the monotonicity property (2.3) shows that

$$
\frac{H_{V}(x, y)}{H_{\Omega}(x, y)} \leq \frac{H_{U}(x, y)}{H_{\tilde{U}}(x, y)} \leq \frac{H_{\Omega}(x, y)}{H_{V}(x, y)} .
$$

Also, because $\Omega^{L} \subset V \subset U, \tilde{U} \subset \Omega$, we see that on the event $\left\{\eta \subset \Omega^{L}\right\}$, we have

$$
-\mu\left(\Omega ; \Omega^{L}, \Omega \backslash V\right) \leq \mu(U ; \eta, U \backslash \tilde{U})-\mu(\tilde{U} ; \eta, \tilde{U} \backslash U) \leq \mu\left(\Omega ; \Omega^{L}, \Omega \backslash V\right) .
$$

These facts imply that $R(\eta) \geq \mathbb{1}_{\left\{\eta \subset \Omega^{L}\right\}} \varepsilon$, where $\varepsilon=\varepsilon\left(\Omega, \Omega^{L}, V\right)>0$ is independent of $U$ and $\tilde{U}$.

Now, denote the probability $\mathbb{P}\left[\eta \subset \Omega^{L}\right]$ by $p$. We conclude that the total variation distance of the law of $\tilde{\eta}$ restricted to $\left\{\tilde{\eta} \subset \Omega^{L}\right\}$ and the law of $\eta$ restricted to $\left\{\eta \subset \Omega^{L}\right\}$ is bounded from above by

$$
\mathbb{E}\left[(1-R(\eta))^{+} \mathbb{1}_{\left\{\eta \subset \Omega^{L}\right\}}\right] \leq p-p \varepsilon .
$$

Thus, there exists a coupling $(\tilde{\eta}, \eta)$ such that $\mathbb{P}\left[\tilde{\eta}=\eta \subset \Omega^{L}\right] \geq p \varepsilon$. From the first part of the proof, we see that $p \geq p_{0}\left(\Omega, \Omega^{L}, V\right)$. This proves the asserted result.

It is important that the bounds in the technical Lemmas 3.5 and 3.6 are uniform over the domains $U$ and $\tilde{U}$. In [28], Lemma 4.2, the authors proved a seemingly similar result, but they only showed that there exists a coupling $(\eta, \tilde{\eta})$ such that $\mathbb{P}[\eta=\tilde{\eta}]>0$, whereas in Lemma 3.6 we proved that $\mathbb{P}[\eta=\tilde{\eta}] \geq \theta$ with the constant $\theta$ uniform over $U$ and $\tilde{U}$.

Let us also emphasize that the assumption in Lemma 3.5 is $\Omega^{L}, U \subset \Omega$, while the assumption in Lemma 3.6 is $\Omega^{L} \subset U \subset \Omega$. Lemma 3.5 is the key point in the proof of the uniqueness in Proposition 3.2, as it guarantees that there is a uniformly positive probability to couple two Markov chains for any initial values. In order to extend the proof of Proposition 3.2 for the range $\kappa \in(4,8)$, Lemma 3.5 has to be extended to this range.

REMARK 3.7. It is also worthwhile to discuss the optimal value of the constant $\theta$ in Lemmas 3.5 and 3.6. When $\kappa \in[8 / 3,4]$, we know this optimal value exactly: namely, from the proof of Lemma 3.5, we see that the optimal constant $\theta=\theta\left(\Omega, \Omega^{L}\right)$ equals $\mathbb{P}\left[\gamma \subset \Omega^{L}\right]$, the probability of the $\operatorname{SLE}_{\kappa}$ curve $\gamma \sim \mathbb{P}(\Omega ; x, y)$ to stay in $\Omega^{L}$. Also, in Lemma 3.6, if $\kappa \in$ $[8 / 3,4]$, then we can use the coupling of Corollary 2.5 , which gives the optimal constant $\theta=$ $\theta\left(\Omega, \Omega^{L}, V\right)=\mathbb{P}\left[\gamma \subset \Omega^{L}\right]$. In particular, this constant does not depend on $V$, so Lemma 3.6 actually holds for all $\Omega^{L} \subset U, \tilde{U} \subset \Omega$.

Now, we are ready to prove Proposition 3.2.

ProOF OF PROPOSITION 3.2. By conformal invariance, it suffices to consider the domain $\Omega=[0, \ell] \times[0,1]$ with marked boundary points $x^{L}=(0,0), x^{R}=(\ell, 0), y^{R}=(\ell, 1)$, $y^{L}=(0,1)$. We define a Markov chain on pairs of curves $\left(\eta^{L}, \eta^{R}\right) \in X_{0}\left(\Omega ; x^{L}, x^{R}, y^{R}, y^{L}\right)$ as follows (see also Figure 3). Given a configuration $\left(\eta_{n}^{L}, \eta_{n}^{R}\right) \in X_{0}\left(\Omega ; x^{L}, x^{R}, y^{R}, y^{L}\right)$, we pick $i \in\{L, R\}$ uniformly and resample $\eta_{n+1}^{i}$ according to the conditional law given the other curve. We will prove that this Markov chain has a unique stationary measure. 

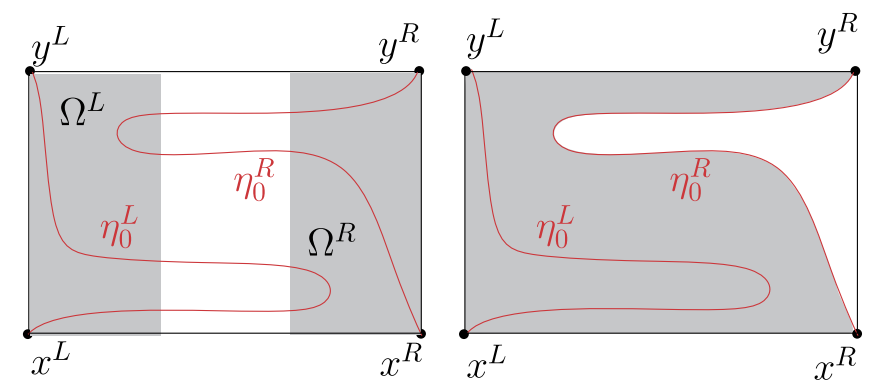

FIG. 3. In the left panel, the two gray parts indicate $\Omega^{L}$ and $\Omega^{R}$ and the two red curves $\eta_{0}^{L}$ and $\eta_{0}^{R}$. In the right panel, given $\eta_{1}^{R}=\eta_{0}^{R}$, we sample $\eta_{1}^{L}$ as the $\mathrm{SLE}_{\kappa}$ in the gray domain between $x^{L}$ and $y^{L}$. Lemma 3.5 guarantees that $\mathbb{P}\left[\eta_{1}^{L} \subset \Omega^{L} \mid \eta_{1}^{R}\right] \geq \theta_{1}$. Then we set $\eta_{2}^{L}=\eta_{1}^{L}$, and hence, $\mathbb{P}\left[\eta_{2}^{L} \subset \Omega^{L}\right] \geq \theta_{1}$.

Take two initial configurations $\left(\eta_{0}^{L}, \eta_{0}^{R}\right)$ and $\left(\tilde{\eta}_{0}^{L}, \tilde{\eta}_{0}^{R}\right)$. We will show that there exists a constant $p_{0}>0$, independent of the initial configurations, and a coupling of $\left(\eta_{4}^{L}, \eta_{4}^{R}\right)$ and $\left(\tilde{\eta}_{4}^{L}, \tilde{\eta}_{4}^{R}\right)$ such that

$$
\mathbb{P}\left[\left(\eta_{4}^{L}, \eta_{4}^{R}\right)=\left(\tilde{\eta}_{4}^{L}, \tilde{\eta}_{4}^{R}\right)\right] \geq p_{0} .
$$

As depicted in Figure 3, we denote $\Omega^{L}=[0, \ell / 3] \times[0,1]$ and $\Omega^{R}=[2 \ell / 3, \ell] \times[0,1]$, and we denote by $\theta_{1}=\theta\left(\Omega, \Omega^{L}\right)=\theta\left(\Omega, \Omega^{R}\right)$ the constant obtained from Lemma 3.5. Given an initial configuration $\left(\eta_{0}^{L}, \eta_{0}^{R}\right) \in X_{0}\left(\Omega ; x^{L}, x^{R}, y^{R}, y^{L}\right)$, we sample $\eta_{1}^{L}$ according to the conditional law and set $\eta_{1}^{R}=\eta_{0}^{R}$. Then we sample $\eta_{2}^{R}$ according to the conditional law and set $\eta_{2}^{L}=\eta_{1}^{L}$. This operation has probability $1 / 4$. Knowing this sampling order, Lemma 3.5 gives (see Figure 3 )

$$
\mathbb{P}\left[\eta_{2}^{L} \subset \Omega^{L}\right] \geq \theta_{1} \quad \text { and } \quad \mathbb{P}\left[\eta_{2}^{R} \subset \Omega^{R} \mid \eta_{2}^{L}\right] \geq \theta_{1}
$$

Thus, for any initial configurations, we have the uniform bound

$$
\mathbb{P}\left[\eta_{2}^{L} \subset \Omega^{L}, \eta_{2}^{R} \subset \Omega^{R}\right] \geq \frac{1}{4} \theta_{1}^{2} .
$$

Now, suppose that we have two initial configurations $\left(\eta_{0}^{L}, \eta_{0}^{R}\right)$ and $\left(\tilde{\eta}_{0}^{L}, \tilde{\eta}_{0}^{R}\right)$, and we sample $\left(\eta_{2}^{L}, \eta_{2}^{R}\right)$ and $\left(\tilde{\eta}_{2}^{L}, \tilde{\eta}_{2}^{R}\right)$ independently. From (3.9), we see that

$$
\mathbb{P}\left[\eta_{2}^{L} \subset \Omega^{L}, \tilde{\eta}_{2}^{L} \subset \Omega^{L}, \eta_{2}^{R} \subset \Omega^{R}, \tilde{\eta}_{2}^{R} \subset \Omega^{R}\right] \geq \frac{1}{16} \theta_{1}^{4}
$$

Then, given $\left(\eta_{2}^{L}, \eta_{2}^{R}, \tilde{\eta}_{2}^{L}, \tilde{\eta}_{2}^{R}\right)$, we resample $\eta_{3}^{L}$ and $\tilde{\eta}_{3}^{L}$ according to the conditional law and set $\eta_{3}^{R}=\eta_{2}^{R}$ and $\tilde{\eta}_{3}^{R}=\tilde{\eta}_{2}^{R}$. Lemma 3.6 guarantees that there exists a coupling such that the probability of the event $\left\{\eta_{3}^{L}=\tilde{\eta}_{3}^{L} \subset \Omega^{L}\right\}$ is at least $\theta_{2}>0$, independently of $\left(\eta_{2}^{L}, \eta_{2}^{R}, \tilde{\eta}_{2}^{L}, \tilde{\eta}_{2}^{R}\right)$ as long as $\left\{\eta_{2}^{R}, \tilde{\eta}_{2}^{R} \subset \Omega^{R}\right\}$. Finally, given $\left(\eta_{3}^{L}, \eta_{3}^{R}, \tilde{\eta}_{3}^{L}, \tilde{\eta}_{3}^{R}\right)$, we resample $\eta_{4}^{R}$ and $\tilde{\eta}_{4}^{R}$ according to the conditional law and set $\eta_{4}^{L}=\eta_{3}^{L}$ and $\tilde{\eta}_{4}^{L}=\tilde{\eta}_{3}^{L}$. Similarly, there exists a coupling such that the probability of $\left\{\eta_{4}^{R}=\tilde{\eta}_{4}^{R} \subset \Omega^{R}\right\}$ is at least $\theta_{2}$ as long as $\left\{\eta_{3}^{L}, \tilde{\eta}_{3}^{L} \subset \Omega^{L}\right\}$. In conclusion, there exists a coupling of $\left(\eta_{4}^{L}, \eta_{4}^{R}\right)$ and $\left(\tilde{\eta}_{4}^{L}, \tilde{\eta}_{4}^{R}\right)$ such that

$$
\mathbb{P}\left[\left(\eta_{4}^{L}, \eta_{4}^{R}\right)=\left(\tilde{\eta}_{4}^{L}, \tilde{\eta}_{4}^{R}\right)\right] \geq \frac{1}{64} \theta_{1}^{4} \theta_{2}^{2} .
$$

This implies the asserted bound (3.8) with $p_{0}=\frac{1}{64} \theta_{1}^{4} \theta_{2}^{2}$.

In conclusion, both the existence and uniqueness of the 2-SLE ${ }_{\kappa}$ now follow from Lemma 3.4 applied to the kernel $P$ realizing four steps of the above Markov chain on $X_{0}\left(\Omega ; x^{L}, x^{R}, y^{R}, y^{L}\right)$ and (3.8) providing the uniform coupling with $\theta=1-p_{0}$. (Furthermore, the Markov chain is mixing, see Remark 3.8.) 
REMARK 3.8. The Markov chain in the proof of Proposition 3.2 is mixing, that is, there exists a coupling between $\left(\eta_{4 n}^{L}, \eta_{4 n}^{R}\right)$ and the global 2-SLE ${ }_{\kappa}\left(\eta^{L}, \eta^{R}\right)$ so that

$$
\mathbb{P}\left[\left(\eta_{4 n}^{L}, \eta_{4 n}^{R}\right) \neq\left(\eta^{L}, \eta^{R}\right)\right] \leq\left(1-p_{0}\right)^{n}
$$

The above proof also works when the conditional laws of $\eta^{R}$ and $\eta^{L}$ are variants of the chordal $\mathrm{SLE}_{\kappa}$. In particular, we use this argument for certain SLE variants in the proof of Theorem 1.2 in Section 3.3.

3.3. Uniqueness: General case. Next, we generalize our proof for the global 2-SLE ${ }_{\kappa}$ to any number $N \geq 3$ of curves, in order to complete the proof of Theorem 1.2. Recall that, for $\alpha \in \mathrm{LP}_{N}$, we denote by $\mathbb{Q}_{\alpha}^{\#}\left(\Omega ; x_{1}, \ldots, x_{2 N}\right)$ the global $N$-SLE $\mathrm{K}_{\kappa}$ probability measures constructed in Section 3.1. In the general case $N \geq 3$, in order to establish the uniform coupling for Lemma 3.4, we use the properties of the measures $\mathbb{Q}_{\alpha}^{\#}\left(\Omega ; x_{1}, \ldots, x_{2 N}\right)$. Therefore, the Markov chain argument does not yield the existence of the stationary measure.

We begin by generalizing Lemma 3.5. By symmetry, we may assume that $\{1,2\} \in \alpha$. This lemma only uses the definition of a global multiple $\mathrm{SLE}_{\kappa}$ and the property from Lemma 3.5 of the chordal $\mathrm{SLE}_{\kappa}$.

LEMMA 3.9. Let $\kappa \in(0,4]$. Let $\left(\Omega ; x_{1}, \ldots, x_{2 N}\right)$ be a polygon and let $\Omega^{L}, U \subset \Omega$ be sub-polygons such that $\Omega^{L}, U$, and $\Omega$ agree in a neighborhood of the arc $\left(x_{1} x_{2}\right)$. Also, let $\left(\eta_{1}, \ldots, \eta_{N}\right)$ be any global $N-\mathrm{SLE}_{\kappa}$ in $\left(U ; x_{1}, \ldots, x_{2 N}\right)$ such that $\eta_{1}$ is the curve connecting $x_{1}$ and $x_{2}$. Then there exists a constant $\theta=\theta\left(\Omega, \Omega^{L}\right)>0$, independent of $U$, such that $\mathbb{P}\left[\eta_{1} \subset \Omega^{L}\right] \geq \theta$.

ProOF. Denote by $\hat{U}_{1}$ the connected component of $U \backslash \bigcup_{j=2}^{N} \eta_{j}$ with $x_{1}$ and $x_{2}$ on its boundary. Then, the conditional law of $\eta_{1}$ given $\hat{U}_{1}$ is the chordal $\mathrm{SLE}_{\kappa}$ in $\hat{U}_{1}$ connecting $x_{1}$ and $x_{2}$. By Lemma 3.5, we have $\mathbb{P}\left[\eta_{1} \subset \Omega^{L} \mid \hat{U}_{1}\right] \geq \theta\left(\Omega, \Omega^{L}\right)$, independently of $\hat{U}_{1}$. Therefore, $\mathbb{P}\left[\eta_{1} \subset \Omega^{L}\right] \geq \theta\left(\Omega, \Omega^{L}\right)$ as well.

To generalize Lemma 3.6, we use the following auxiliary result, which says that all of the curves have a positive probability to stay in a subdomain of $\Omega$, uniformly with respect to a larger subdomain. Its proof uses the explicit construction of the global $N-\mathrm{SLE}_{\kappa}$ measure presented in Section 3.1.

LEMMA 3.10. Let $\kappa \in(0,4]$. Let $\left(\Omega ; x_{1}, \ldots, x_{2 N}\right)$ be a polygon and $\Omega^{L} \subset U \subset \Omega$ sub-polygons. Suppose $\left(\eta_{1}, \ldots, \eta_{N}\right) \sim \mathbb{Q}_{\alpha}^{\#}\left(U ; x_{1}, \ldots, x_{2 N}\right)$. Then there exists a constant $\theta=\theta\left(\Omega, \Omega^{L}\right)>0$, independent of $U$, such that $\mathbb{P}\left[\eta_{j} \subset \Omega^{L} \forall j\right] \geq \theta$.

PROOF. We prove the lemma separately for $\kappa \in(0,8 / 3]$ and $\kappa \in[8 / 3,4]$. Assume first that $\kappa \in(0,8 / 3]$. Let $\left(\gamma_{1}^{L}, \ldots, \gamma_{N}^{L}\right)$ be sampled according to $\mathbb{Q}_{\alpha}^{\#}\left(\Omega^{L} ; x_{1}, \ldots, x_{2 N}\right)$. By Proposition 3.1 , we have

$$
\mathbb{P}\left[\eta_{j} \subset \Omega^{L} \forall j\right]=\frac{\mathcal{Z}_{\alpha}\left(\Omega^{L} ; x_{1}, \ldots, x_{2 N}\right)}{\mathcal{Z}_{\alpha}\left(U ; x_{1}, \ldots, x_{2 N}\right)} \mathbb{E}\left[\exp \left(-c \mu\left(U ; U \backslash \Omega^{L}, \bigcup_{j=1}^{N} \gamma_{j}^{L}\right)\right)\right] .
$$

Since $\kappa \leq 8 / 3$, we have $c \leq 0$ by (2.5). Thus, combining with the monotonicity property (3.4), we obtain

$$
\mathbb{P}\left[\eta_{j} \subset \Omega^{L} \forall j\right] \geq \frac{\mathcal{Z}_{\alpha}\left(\Omega^{L} ; x_{1}, \ldots, x_{2 N}\right)}{\mathcal{Z}_{\alpha}\left(U ; x_{1}, \ldots, x_{2 N}\right)} \geq \frac{\mathcal{Z}_{\alpha}\left(\Omega^{L} ; x_{1}, \ldots, x_{2 N}\right)}{\mathcal{Z}_{\alpha}\left(\Omega ; x_{1}, \ldots, x_{2 N}\right)}>0,
$$

where the lower bound is independent of $U$, as claimed. 
Assume next that $\kappa \in[8 / 3,4]$. Let $\left(\gamma_{1}, \ldots, \gamma_{N}\right) \sim \mathbb{Q}_{\alpha}^{\#}\left(\Omega ; x_{1}, \ldots, x_{2 N}\right)$. By Proposition 3.1 , we have

$$
\mathbb{P}\left[\eta_{j} \subset \Omega^{L} \forall j\right]=\frac{\mathcal{Z}_{\alpha}\left(\Omega ; x_{1}, \ldots, x_{2 N}\right)}{\mathcal{Z}_{\alpha}\left(U ; x_{1}, \ldots, x_{2 N}\right)} \mathbb{E}\left[\mathbb{1}_{\left\{\forall j, \gamma_{j} \subset U\right\}} \exp \left(c \mu\left(\Omega ; \Omega \backslash U, \bigcup_{j=1}^{N} \gamma_{j}\right)\right)\right]
$$

Since $\kappa \in[8 / 3,4]$, we have $c \geq 0$ by $(2.5)$, so we obtain

$$
\begin{aligned}
\mathbb{P}\left[\eta_{j} \subset \Omega^{L} \forall j\right] & \geq \frac{\mathcal{Z}_{\alpha}\left(\Omega ; x_{1}, \ldots, x_{2 N}\right)}{\mathcal{Z}_{\alpha}\left(U ; x_{1}, \ldots, x_{2 N}\right)} \mathbb{P}\left[\gamma_{j} \subset \Omega^{L} \forall j\right] \\
& \geq \frac{\mathcal{Z}_{\alpha}\left(\Omega ; x_{1}, \ldots, x_{2 N}\right)}{\prod_{\{a, b\} \in \alpha} H_{U}\left(x_{a}, x_{b}\right)^{h}} \mathbb{P}\left[\gamma_{j} \subset \Omega^{L} \forall j\right] \quad \text { [by (3.3)] } \\
& \geq \frac{\mathcal{Z}_{\alpha}\left(\Omega ; x_{1}, \ldots, x_{2 N}\right)}{\prod_{\{a, b\} \in \alpha} H_{\Omega}\left(x_{a}, x_{b}\right)^{h}} \mathbb{P}\left[\gamma_{j} \subset \Omega^{L} \forall j\right]>0 \quad \text { [by (2.3)]. }
\end{aligned}
$$

This gives the assertion for $\kappa \in[8 / 3,4]$ and completes the proof.

Now, we prove an analogue of Lemma 3.6 for $\kappa \leq 4$ and $N \geq 3$, using the explicit construction of the global $N-\mathrm{SLE}_{\kappa}$ measure presented in Section 3.1.

LEMMA 3.11. Let $\kappa \in(0,4]$. Let $\left(\Omega ; x_{1}, \ldots, x_{2 N}\right)$ be a polygon, and let $\Omega^{L} \subset V \subset U$ and $\tilde{U} \subset \Omega$ be subpolygons such that $\operatorname{dist}\left(\Omega^{L}, \Omega \backslash V\right)>0$. Also, suppose that $\left(\eta_{1}, \ldots, \eta_{N}\right) \sim$ $\mathbb{Q}_{\alpha}^{\#}\left(U ; x_{1}, \ldots, x_{2 N}\right)$ and $\left(\tilde{\eta}_{1}, \ldots, \tilde{\eta}_{N}\right) \sim \mathbb{Q}_{\alpha}^{\#}\left(\tilde{U} ; x_{1}, \ldots, x_{2 N}\right)$. Then there exists a coupling of $\left(\eta_{1}, \ldots, \eta_{N}\right)$ and $\left(\tilde{\eta}_{1}, \ldots, \tilde{\eta}_{N}\right)$ such that $\mathbb{P}\left[\eta_{j}=\tilde{\eta}_{j} \subset \Omega^{L} \forall j\right] \geq \theta$, where the constant $\theta=\theta\left(\Omega, \Omega^{L}, V\right)>0$ is independent of $U$ and $\tilde{U}$.

ProOF. By Proposition 3.1, the law of $\left(\tilde{\eta}_{1}, \ldots, \tilde{\eta}_{N}\right)$ restricted to $\left\{\tilde{\eta}_{j} \subset \Omega^{L} \forall j\right\}$ is absolutely continuous with respect to the law of $\left(\eta_{1}, \ldots, \eta_{N}\right)$ restricted to $\left\{\eta_{j} \subset \Omega^{L} \forall j\right\}$, with Radon-Nikodym derivative

$$
\begin{aligned}
R\left(\eta_{1}, \ldots, \eta_{N}\right)= & \frac{\mathcal{Z}_{\alpha}\left(U ; x_{1}, \ldots, x_{2 N}\right)}{\mathcal{Z}_{\alpha}\left(\tilde{U} ; x_{1}, \ldots, x_{2 N}\right)} \mathbb{1}_{\left\{\eta_{j} \subset \Omega^{L} \forall j\right\}} \\
& \times \exp \left(c \mu\left(U ; U \backslash \Omega^{L}, \bigcup_{j=1}^{N} \eta_{j}\right)-c \mu\left(\tilde{U} ; \tilde{U} \backslash \Omega^{L}, \bigcup_{j=1}^{N} \eta_{j}\right)\right) .
\end{aligned}
$$

First, we will find a positive lower bound for $R\left(\eta_{1}, \ldots, \eta_{N}\right)$, separately for $\kappa \in(0,8 / 3]$ and $\kappa \in[8 / 3,4]$. Since $\Omega^{L} \subset V \subset U, \tilde{U} \subset \Omega$, on the event $\left\{\eta_{j} \subset \Omega^{L} \forall j\right\}$, we have

$$
\left|\mu\left(U ; U \backslash \Omega^{L}, \bigcup_{j=1}^{N} \eta_{j}\right)-\mu\left(\tilde{U} ; \tilde{U} \backslash \Omega^{L}, \bigcup_{j=1}^{N} \eta_{j}\right)\right| \leq \mu\left(\Omega ; \Omega \backslash V, \Omega^{L}\right) .
$$

When $\kappa \in(0,8 / 3]$, we have $c \leq 0$ by (2.5). Thus, using the monotonicity property (3.4), we see that on the event $\left\{\eta_{j} \subset \Omega^{L} \forall j\right\}$, we have

$$
R\left(\eta_{1}, \ldots, \eta_{N}\right) \geq \frac{\mathcal{Z}_{\alpha}\left(\Omega^{L} ; x_{1}, \ldots, x_{2 N}\right)}{\mathcal{Z}_{\alpha}\left(\Omega ; x_{1}, \ldots, x_{2 N}\right)} \exp \left(c \mu\left(\Omega ; \Omega \backslash V, \Omega^{L}\right)\right)>0 .
$$

On the other hand, when $\kappa \in[8 / 3,4]$, we have $c \geq 0$ by (2.5). On the event $\left\{\eta_{j} \subset \Omega^{L} \forall j\right\}$, we have

$$
R\left(\eta_{1}, \ldots, \eta_{N}\right) \geq \frac{\mathcal{Z}_{\alpha}\left(U ; x_{1}, \ldots, x_{2 N}\right)}{\mathcal{Z}_{\alpha}\left(\tilde{U} ; x_{1}, \ldots, x_{2 N}\right)} \exp \left(-c \mu\left(\Omega ; \Omega \backslash V, \Omega^{L}\right)\right)
$$


Using (3.3) and (2.3), we estimate the denominator as

$$
\mathcal{Z}_{\alpha}\left(\tilde{U} ; x_{1}, \ldots, x_{2 N}\right) \leq \prod_{\{a, b\} \in \alpha} H_{\tilde{U}}\left(x_{a}, x_{b}\right)^{h} \leq \prod_{\{a, b\} \in \alpha} H_{\Omega}\left(x_{a}, x_{b}\right)^{h},
$$

and using (2.3), we estimate the numerator as

$$
\begin{aligned}
\mathcal{Z}_{\alpha}\left(U ; x_{1}, \ldots, x_{2 N}\right) & =\prod_{\{a, b\} \in \alpha} H_{U}\left(x_{a}, x_{b}\right)^{h} \times f_{\alpha}\left(U ; x_{1}, \ldots, x_{2 N}\right) \\
& \geq \prod_{\{a, b\} \in \alpha} H_{\Omega^{L}}\left(x_{a}, x_{b}\right)^{h} \times f_{\alpha}\left(U ; x_{1}, \ldots, x_{2 N}\right) .
\end{aligned}
$$

Taking the infimum over all subpolygons $A$ such that $V \subset A \subset \Omega$, we have

$$
f_{\alpha}\left(U ; x_{1}, \ldots, x_{2 N}\right) \geq \inf _{A} f_{\alpha}\left(A ; x_{1}, \ldots, x_{2 N}\right):=v(\Omega, V) .
$$

We next show that this infimum is strictly positive. By conformal invariance of $f_{\alpha}$, we may take $\Omega=\mathbb{H}$, and we have

$$
f_{\alpha}\left(A ; x_{1}, \ldots, x_{2 N}\right)=f_{\alpha}\left(\mathbb{H} ; \varphi_{A}\left(x_{1}\right), \ldots, \varphi_{A}\left(x_{2 N}\right)\right)>0
$$

for any conformal map $\varphi_{A}: A \rightarrow \mathbb{H}$. Now, we have

$$
v(\Omega, V)=\inf _{\left(y_{1}, \ldots, y_{2 N}\right) \in K} f_{\alpha}\left(\mathbb{H} ; y_{1}, \ldots, y_{2 N}\right)>0,
$$

where $K$ is a compact subset of $\mathbb{R}^{2 N}$ such that $\left(\varphi_{A}\left(x_{1}\right), \ldots, \varphi_{A}\left(x_{2 N}\right)\right) \in K$ for all $A$. Thus, we obtain

$$
\mathcal{Z}_{\alpha}\left(U ; x_{1}, \ldots, x_{2 N}\right) \geq \prod_{\{a, b\} \in \alpha} H_{\Omega^{L}}\left(x_{a}, x_{b}\right)^{h} \times v(\Omega, V)>0 .
$$

After combining (3.11) and (3.12), we finally obtain

$$
R\left(\eta_{1}, \ldots, \eta_{N}\right) \geq \prod_{\{a, b\} \in \alpha}\left(\frac{H_{\Omega^{L}}\left(x_{a}, x_{b}\right.}{H_{\Omega}\left(x_{a}, x_{b}\right.}\right)^{h} v(\Omega, V) e^{-c \mu\left(\Omega ; \Omega \backslash V, \Omega^{L}\right)}>0 .
$$

In both estimates (3.10) and (3.13), we obtain a lower bound $R\left(\eta_{1}, \ldots, \eta_{N}\right) \geq \varepsilon:=$ $\varepsilon\left(\Omega, \Omega^{L}, V\right)>0$, independently of $U$ and $\tilde{U}$, as desired. This completes the first part of the proof.

Now, denote the probability $\mathbb{P}\left[\eta_{j} \subset \Omega^{L} \forall j\right]$ by $p$. The total variation distance of the law of $\left(\tilde{\eta}_{1}, \ldots, \tilde{\eta}_{N}\right)$ restricted to $\left\{\tilde{\eta}_{j} \subset \Omega^{L} \forall j\right\}$ and the law of $\left(\eta_{1}, \ldots, \eta_{N}\right)$ restricted to $\left\{\eta_{j} \subset\right.$ $\left.\Omega^{L} \forall j\right\}$ is bounded from above by

$$
\mathbb{E}\left[\left(1-R\left(\eta_{1}, \ldots, \eta_{N}\right)\right)^{+} \mathbb{1}_{\left\{\eta_{j} \subset \Omega^{L} \forall j\right\}}\right] \leq p(1-\varepsilon) .
$$

It follows from this observation that there exists a coupling of $\left(\eta_{1}, \ldots, \eta_{N}\right)$ and $\left(\tilde{\eta}_{1}, \ldots, \tilde{\eta}_{N}\right)$ such that $\mathbb{P}\left[\tilde{\eta}_{j}=\eta_{j} \subset \Omega^{L} \forall j\right] \geq p \varepsilon$. Combining this with Lemma 3.10, we obtain the asserted result.

We are now ready to conclude with the proof of Theorem 1.2.

ProOf OF THEOREM 1.2. The existence was proved in [21, 25, 31], and summarized in Section 3.1. Hence, we only need to prove the uniqueness. To this end, we proceed by induction on $N \geq 2$. The case $N=2$ is the content of Corollary 3.3. Thus, we let $N \geq$ 3 and assume that, for any link pattern $\beta \in \mathrm{LP}_{N-1}$, there exists a unique global $(N-1)$ $\mathrm{SLE}_{\kappa}$ associated to $\beta$. For $j \in\{1, \ldots, N-1\}$, we denote by $\mathbb{Q}_{\beta}^{\left\{a_{j}, b_{j}\right\}}\left(\Omega ; x_{1}, \ldots, x_{2 N-2}\right)$ the marginal law of $\eta_{j}$ in this global multiple SLE. 

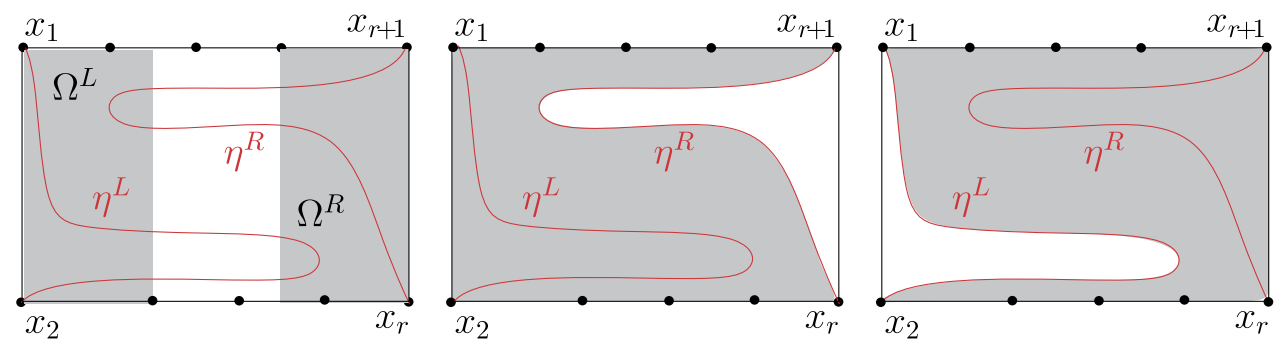

FIG. 4. In the left panel, the two red curves are $\eta^{L}$ and $\eta^{R}$, and the two gray parts are $\Omega^{L}$ and $\Omega^{R}$. In the middle panel, the gray part is the domain $D^{L}$, and the marked points along the boundary of $D^{L}$ are $x_{1}, \ldots, x_{r-1}, x_{r+2}, \ldots, x_{2 N}$. In the right panel, the gray part is the domain $D^{R}$, and the marked points along the boundary of $D^{R}$ are $x_{3}, \ldots, x_{2 N}$.

Now, let $\alpha \in \operatorname{LP}_{N}$ and suppose that $\left(\eta_{1}, \ldots, \eta_{N}\right) \in X_{0}^{\alpha}\left(\Omega ; x_{1}, \ldots, x_{2 N}\right)$ has the law of a global $N-\mathrm{SLE}_{\kappa}$ associated to $\alpha$. By symmetry, we may assume that $\{1,2\},\{r, r+1\} \in \alpha$ with $r \in\{3,4, \ldots, 2 N-1\}$. Denote by $\eta^{L}$ (resp., $\eta^{R}$ ) the curve in the collection $\left\{\eta_{1}, \ldots, \eta_{N}\right\}$ that connects $x_{1}$ and $x_{2}$ (resp., $x_{r}$ and $x_{r+1}$ ). It follows from the induction hypothesis that, given $\left(\eta^{L}, \eta^{R}\right)$, the conditional law of the other $(N-2)$ curves is the unique global $(N-2)-\mathrm{SLE}_{\kappa}$ associated to $(\alpha /\{r, r+1\}) /\{1,2\}$ in the appropriate remaining domain (recalling the link removal notation). Thus, it is sufficient to prove the uniqueness of the joint law on the pair $\left(\eta^{L}, \eta^{R}\right)$.

The induction hypothesis also implies that, given $\eta^{R}$ (resp. $\eta^{L}$ ), the conditional law of the rest of the curves is the unique global $(N-1)-\mathrm{SLE}_{\kappa}$ associated to the link pattern $\alpha /\{r, r+$ $1\}$ (resp., $\alpha /\{1,2\})$. As illustrated in Figure 4, we denote by $D^{L}$ (resp. $D^{R}$ ) the connected component of $\Omega \backslash \eta^{R}$ (resp. $\Omega \backslash \eta^{L}$ ) with $x_{1}$ and $x_{2}$ (resp., $x_{r}$ and $x_{r+1}$ ) on its boundary. Then the conditional law of $\eta^{L}$ given $\eta^{R}$ is

$$
\mathbb{Q}_{\alpha /\{r, r+1\}}^{\{1,2\}}\left(D^{L} ; x_{1}, \ldots, x_{r-1}, x_{r+2}, \ldots, x_{2 N}\right)
$$

and the conditional law of $\eta^{R}$ given $\eta^{L}$ is

$$
\mathbb{Q}_{\alpha /\{1,2\}}^{\{r-2, r-1\}}\left(D^{R} ; x_{3}, \ldots, x_{2 N}\right) \text {. }
$$

Now to finish, following the idea of the proof of Proposition 3.2, we consider Markov chains sampling $\eta^{L}$ and $\eta^{R}$ from these conditional laws. After replacing Lemma 3.5 by Lemma 3.9 (for $N-1$ ) and Lemma 3.6 by Lemma 3.11 (also for $N-1$ ) in the proof of Proposition 3.2, we see that this Markov chain has a unique stationary measure which coincides with the one presented in Section 3.1.

3.4. Marginal law. To conclude this section, we determine the marginal law of a single curve in the global multiple $\mathrm{SLE}_{\kappa}$. Recall that the pure partition functions $\mathcal{Z}_{\alpha}$ were defined in (3.2). We denote

$$
\mathcal{Z}_{\alpha}\left(x_{1}, \ldots, x_{2 N}\right):=\mathcal{Z}_{\alpha}\left(\mathbb{H} ; x_{1}, \ldots, x_{2 N}\right) \text { for } x_{1}<\cdots<x_{2 N} .
$$

Lemma 3.12 ([31], Proposition 4.9). Let $\kappa \in(0,4]$ and $\alpha \in \operatorname{LP}_{N}$. Assume that $\{a, b\} \in$ $\alpha$. Let $W_{t}$ be the solution to the following SDEs:

$$
\begin{aligned}
\mathrm{d} W_{t} & =\sqrt{\kappa} \mathrm{d} B_{t}+\kappa \partial_{a} \log \mathcal{Z}_{\alpha}\left(V_{t}^{1}, \ldots, V_{t}^{a-1}, W_{t}, V_{t}^{a+1}, \ldots, V_{t}^{2 N}\right) \mathrm{d} t, \\
\mathrm{~d} V_{t}^{i} & =\frac{2 \mathrm{~d} t}{V_{t}^{i}-W_{t}}
\end{aligned}
$$


with $W_{0}=x_{a}$ and $V_{0}^{i}=x_{i}$ for $i \neq a$. Then the Loewner chain driven by $W_{t}$ is well-defined up to the swallowing time $T_{b}$ of $x_{b}$. Moreover, it is almost surely generated by a continuous curve up to and including $T_{b}$. This curve has the same law as the one connecting $x_{a}$ and $x_{b}$ in the global multiple $\mathrm{SLE}_{\kappa}$ associated to $\alpha$ in the polygon ( $\left.\mathbb{H} ; x_{1}, \ldots, x_{2 N}\right)$.

4. Multiple interfaces in Ising and random-cluster models. In this final section, we give examples of discrete models whose interfaces converge in the scaling limit to multiple SLEs. More precisely, we consider the critical Ising and random-cluster models in the plane.

In Sections 4.1-4.3, we consider interfaces in the critical random-cluster models with alternating boundary conditions and fixing the connectivity pattern of the curves. We show that, given the convergence of a single interface, multiple interfaces also have a conformally invariant scaling limit, namely the unique global multiple $\operatorname{SLE}_{\kappa}$ with $\kappa \in(4,6]$. Interestingly, this range of the parameter $\kappa$ is beyond the range $(0,4]$, where global multiple SLEs have been explicitly constructed and classified. Thus, from the convergence of these discrete interfaces we would in fact get the existence and uniqueness of the global multiple $\mathrm{SLE}_{\kappa}$ with $\kappa \in(4,6]$. Unfortunately, the convergence of a single interface in the random-cluster model towards the chordal $\mathrm{SLE}_{\kappa}$ has only been rigorously established for the case of $\kappa=16 / 3-$ the FK-Ising model. This is the case appearing in Proposition 1.4, whose proof is completed in Section 4.3. The convergence of two interfaces of the FK-Ising model was also proved in [20], where the authors used a discrete holomorphic observable constructed in [7, 39, 40]. In contrast, our method gives the convergence for any given number of interfaces via a global approach.

In the case of the critical Ising model with alternating boundary conditions, K. Izyurov proved that the collection of any number $N$ of interfaces converges to a multiple SLE process in a local sense [15]. In the present article, we condition the interfaces to forming a given connectivity pattern and prove the convergence of the interfaces as a whole global collection of curves, which we know by Theorem 1.2 to be given by the unique global $N-\mathrm{SLE}_{3}$. This is the content of Section 4.4, where we prove Proposition 1.3. We are also able to determine the marginal law of one curve in this scaling limit. The case of two curves was considered in [43]: in this case, the marginal law is also called a hypergeometric SLE.

In [31], Sections 5 and 6, the authors discussed multiple level lines of the Gaussian free field with alternating boundary data. These level lines give rise to global multiple $\mathrm{SLE}_{4}$ curves (with any connectivity pattern). In this particular case, the marginal law of one curve in the global multiple $\mathrm{SLE}_{4}$ degenerates to a certain $\operatorname{SLE}_{4}(\rho)$ process. In general, however, the marginal laws of single curves in global multiple SLEs are not $\operatorname{SLE}_{\kappa}(\rho)$ processes, but certain more general variants of the chordal $\mathrm{SLE}_{\kappa}$. We refer to [31], Section 3, for more details.

Notation and terminology. We will use the following notions throughout. For notational simplicity, we only consider the square lattice $\mathbb{Z}^{2}$. Two vertices $v$ and $w$ are said to be neighbors if their Euclidean distance equals one, which we denote by $v \sim w$. For a finite subgraph $\mathcal{G}=(V(\mathcal{G}), E(\mathcal{G}))$ of $\mathbb{Z}^{2}$, we denote by $\partial \mathcal{G}$ the inner boundary of $\mathcal{G}:$

$$
\partial \mathcal{G}=\left\{v \in V(\mathcal{G}): \exists w \notin V(\mathcal{G}) \text { such that }\langle v, w\rangle \in E\left(\mathbb{Z}^{2}\right)\right\} .
$$

As an abuse of notation, we sometimes let $\mathcal{G}$ also denote the simply connected domain formed by all of the faces, edges and vertices of $\mathcal{G}$.

In the case of the square lattice, the dual lattice $\left(\mathbb{Z}^{2}\right)^{*}$ is just a translated version of $\mathbb{Z}^{2}$. More precisely, $\left(\mathbb{Z}^{2}\right)^{*}$ is the dual graph of $\mathbb{Z}^{2}$ : its vertex set is $(1 / 2,1 / 2)+\mathbb{Z}^{2}$ and its edges are given by all pairs $\left(v_{1}, v_{2}\right)$ of vertices that are neighbors. The vertices and edges of $\left(\mathbb{Z}^{2}\right)^{*}$ are called dual-vertices and dual-edges. In particular, for each edge $e$ of $\mathbb{Z}^{2}$, we associate a dual-edge, denoted by $e^{*}$, that crosses $e$ in the middle. For a subgraph $\mathcal{G}$ of $\mathbb{Z}^{2}$, we define $\mathcal{G}^{*}$ 
to be the subgraph of $\left(\mathbb{Z}^{2}\right)^{*}$ with edge set $E\left(\mathcal{G}^{*}\right)=\left\{e^{*}: e \in E(\mathcal{G})\right\}$ and vertex set given by the endpoints of these dual-edges.

Finally, the medial lattice $\left(\mathbb{Z}^{2}\right)^{\diamond}$ is the graph with the centers of edges of $\mathbb{Z}^{2}$ as the vertex set, and edges given by all pairs of vertices that are neighbors. In the case of the square lattice, the medial lattice is a rotated and rescaled version of $\mathbb{Z}^{2}$. We identify the faces of $\left(\mathbb{Z}^{2}\right)^{\diamond}$ with the vertices of $\mathbb{Z}^{2}$ and $\left(\mathbb{Z}^{2}\right)^{*}$.

Now, suppose that $\mathcal{G}$ is a finite connected subgraph of the (possibly translated, rotated and rescaled) square lattice $\mathbb{Z}^{2}$ such that the complement of $\mathcal{G}$ is also connected (this means that $\mathcal{G}$ is simply connected). Then we call a triple $(\mathcal{G} ; v, w)$ with $v, w \in \partial \mathcal{G}$ distinct boundary vertices a discrete Dobrushin domain. We note that the boundary $\partial \mathcal{G}$ is divided into two $\operatorname{arcs}(v w)$ and $(w v)$. More generally, given distinct boundary vertices $v_{1}, \ldots, v_{2 N} \in \partial \mathcal{G}$ in counterclockwise order, we call the $(2 N+1)$-tuple $\left(\mathcal{G} ; v_{1}, \ldots, v_{2 N}\right)$ a discrete polygon. In this case, the boundary $\partial \mathcal{G}$ is divided into $2 N$ arcs.

In this article, we consider scaling limits of models on discrete lattices with mesh size tending to zero. We only consider the following square lattice approximations, even though the results discussed in this section hold in a more general setting as well; see [7]. For small $\delta>0$, we let $\Omega^{\delta}$ denote a finite subgraph of the rescaled square lattice $\delta \mathbb{Z}^{2}$. Like $\Omega^{\delta}$, we decorate its vertices and edges with the mesh size $\delta$ as a superscript. The definitions of the dual lattice $\Omega_{*}^{\delta}:=\left(\Omega^{\delta}\right)^{*}$, the medial lattice $\Omega_{\diamond}^{\delta}:=\left(\Omega^{\delta}\right)^{\diamond}$ and discrete Dobrushin domains and polygons obviously extend to this context.

DEFINITION 4.1. Let $\left(\Omega ; x_{1}, \ldots, x_{2 N}\right)$ be a bounded polygon and consider a sequence $\left(\left(\Omega^{\delta} ; x_{1}^{\delta}, \ldots, x_{2 N}^{\delta}\right)\right)_{\delta>0}$ of discrete polygons. We say that $\left(\Omega^{\delta} ; x_{1}^{\delta}, \ldots, x_{2 N}^{\delta}\right)$ converges to $\left(\Omega ; x_{1}, \ldots, x_{2 N}\right)$ as $\delta \rightarrow 0$ in the Carathéodory sense if there exist conformal maps $f^{\delta}$ (resp. $f$ ) from the unit disc $\mathbb{U}=\{z \in \mathbb{C}:|z|<1\}$ to $\Omega^{\delta}$ (resp., from $\mathbb{U}$ to $\Omega$ ) such that $f^{\delta} \rightarrow f$ uniformly on any compact subset of $\mathbb{U}$, and for all $j \in\{1, \ldots, 2 N\}$, we have $\lim _{\delta \rightarrow 0}\left(f^{\delta}\right)^{-1}\left(x_{j}^{\delta}\right)=f^{-1}\left(x_{j}\right)$.

4.1. Random-cluster models. Let $\mathcal{G}=(V(\mathcal{G}), E(\mathcal{G}))$ be a finite subgraph of $\mathbb{Z}^{2}$. A (percolation) configuration $\omega=\left(\omega_{e}\right)_{e \in E(\mathcal{G})}$ is an element of $\{0,1\}^{E(\mathcal{G})}$. If $\omega_{e}=1$, the edge $e$ is said to be open, and otherwise, $e$ is said to be closed. The configuration $\omega$ can be seen as a subgraph of $\mathcal{G}$ with the same set of vertices $V(\mathcal{G})$ and whose edges are the open edges $\left\{e \in E(\mathcal{G}): \omega_{e}=1\right\}$. We denote by $o(\omega)$ (resp., $\left.c(\omega)\right)$ the number of open (resp., closed) edges of $\omega$.

We are interested in the connectivity properties of the graph $\omega$. The maximal connected components of $\omega$ are called clusters. Two vertices $u$ and $v$ are connected by $\omega$ inside $S \subset \mathbb{Z}^{2}$ if there exists a sequence $\left\{v_{j}: 0 \leq j \leq k\right\}$ of vertices in $S$ such that $v_{0}=u, v_{k}=v$, and each edge $\left\langle v_{j}, v_{j+1}\right\rangle$ is open in $\omega$ for $0 \leq j<k$.

We may also impose to our model various boundary conditions, which can be understood as encoding how the sites are connected outside $\mathcal{G}$. A boundary condition $\xi$ is a partition $P_{1} \sqcup \cdots \sqcup P_{k}$ of $\partial \mathcal{G}$. Two vertices are said to be wired in $\xi$ if they belong to the same $P_{j}$ and free otherwise. We denote by $\omega^{\xi}$ the (quotient) graph obtained from the configuration $\omega$ by identifying the wired vertices together in $\xi$.

The probability measure $\phi_{p, q, \mathcal{G}}^{\xi}$ of the random-cluster model on $\mathcal{G}$ with edge-weight $p \in$ $[0,1]$, cluster-weight $q>0$, and boundary condition $\xi$, is defined by

$$
\begin{aligned}
\phi_{p, q, \Omega}^{\xi}[\omega] & :=\frac{p^{o(\omega)}(1-p)^{c(\omega)} q^{k\left(\omega^{\xi}\right)}}{Z_{p, q, \Omega}^{\xi}}, \\
\text { where } Z_{p, q, \Omega}^{\xi} & =\sum_{\omega} p^{o(\omega)}(1-p)^{c(\omega)} q^{k\left(\omega^{\xi}\right)},
\end{aligned}
$$


where and $k\left(\omega^{\xi}\right)$ is the number of connected components of the graph $\omega^{\xi}$. For $q=1$, this model is simply the Bernoulli bond percolation. For $q=2$, the random-cluster model is also known as the FK-Ising model, closely related to the spin Ising model. Proposition 1.4 concerns this case.

For a configuration $\xi$ on $E\left(\mathbb{Z}^{2}\right) \backslash E(\mathcal{G})$, the boundary condition induced by $\xi$ is defined as the partition $P_{1} \sqcup \cdots \sqcup P_{k}$, where two vertices belong to the same $P_{j}$ if and only if there exists an open path in $\xi$ connecting them. We identify the boundary condition induced by $\xi$ with the configuration itself, and denote the measure of the random-cluster model with such boundary conditions by $\phi_{p, q, \mathcal{G}}^{\xi}$. As a direct consequence of these definitions, we have the following domain Markov property. Suppose that $\mathcal{G} \subset \mathcal{G}^{\prime}$ are two finite subgraphs of $\mathbb{Z}^{2}$. Fix $p \in[0,1], q>0$, and a boundary condition $\xi$ on $\partial \mathcal{G}^{\prime}$. Let $X$ be a random variable, which is measurable with respect to the status of the edges in $\mathcal{G}$. Then, for all $\psi \in\{0,1\}^{E\left(\mathcal{G}^{\prime}\right) \backslash E(\mathcal{G})}$, we have

$$
\phi_{p, q, \mathcal{G}^{\prime}}^{\xi}\left[X \mid \omega_{e}=\psi_{e} \text { for all } e \in E\left(\mathcal{G}^{\prime}\right) \backslash E(\mathcal{G})\right]=\phi_{p, q, \mathcal{G}}^{\psi^{\xi}}[X],
$$

where $\psi^{\xi}$ is the partition on $\partial \mathcal{G}$ obtained by wiring two vertices in $\partial \mathcal{G}$ if they are connected in $\psi$.

We define an ordering for configurations as follows. For $\omega, \omega^{\prime} \in\{0,1\}^{E(\mathcal{G})}$, we denote by $\omega \leq \omega^{\prime}$ if $\omega_{e} \leq \omega_{e}^{\prime}$ for all $e \in E(\mathcal{G})$. An event $\mathcal{A}$ depending on the edges in $E(\mathcal{G})$ is said to be increasing if, for any $\omega \in \mathcal{A}$, the inequality $\omega \leq \omega^{\prime}$ implies that $\omega^{\prime} \in \mathcal{A}$. When $q \geq 1$, the following FKG inequality (positive association) holds. Fix $p \in[0,1], q \geq 1$, and a boundary condition $\xi$ on $\partial \mathcal{G}$. Then, for any two increasing events $\mathcal{A}$ and $\mathcal{B}$, we have

$$
\phi_{p, q, \mathcal{G}}^{\xi}[\mathcal{A} \cap \mathcal{B}] \geq \phi_{p, q, \mathcal{G}}^{\xi}[\mathcal{A}] \phi_{p, q, \mathcal{G}}^{\xi}[\mathcal{B}] .
$$

Consequently, for any boundary conditions $\xi \leq \psi$ and for any increasing event $\mathcal{A}$, we have

$$
\phi_{p, q, \mathcal{G}}^{\xi}[\mathcal{A}] \leq \phi_{p, q, \mathcal{G}}^{\psi}[\mathcal{A}] .
$$

We denote by $\phi_{p, q, \mathcal{G}}^{0}$ the probability measure of the random-cluster model with free boundary conditions, where the partition $\xi$ of $\partial \mathcal{G}$ consists of singletons only. We denote by $\phi_{p, q, \mathcal{G}}^{1}$ the probability measure of the random-cluster model with wired boundary conditions, where the partition $\xi$ of $\partial \mathcal{G}$ is the whole set $\partial \mathcal{G}$. In the sense of $(4.1), \phi_{p, q, \mathcal{G}}^{0}$ is minimal and $\phi_{p, q, \mathcal{G}}^{1}$ is maximal.

A configuration $\omega$ on $\mathcal{G}$ can be uniquely associated to a dual configuration $\omega^{*}$ on the dual graph $\mathcal{G}^{*}$, defined by $\omega^{*}\left(e^{*}\right)=1-\omega(e)$ for all $e \in E(\mathcal{G})$. A dual-edge $e^{*}$ is said to be dualopen if $\omega^{*}\left(e^{*}\right)=1$ and dual-closed otherwise. A dual-cluster is a connected component of $\omega^{*}$. We extend the notions of dual-open paths and connectivity events in the obvious way. Now, if $\omega$ is distributed according to $\phi_{p, q, \mathcal{G}}^{\xi}$, then $\omega^{*}$ is distributed according to $\phi_{p^{*}, q^{*}, \mathcal{G}^{*}}^{\xi^{*}}$, with

$$
q^{*}=q \quad \text { and } \quad \frac{p p^{*}}{(1-p)\left(1-p^{*}\right)}=q .
$$

Note that, at the self-dual point $p^{*}=p$, we have

$$
p=p_{c}(q):=\frac{\sqrt{q}}{1+\sqrt{q}} .
$$

For this critical case $p=p_{c}(q)$, we have the following Russo-Seymour-Welsh (RSW) estimate. For a rectangle $R=[a, b] \times[c, d] \subset \mathbb{Z}^{2}$, we let $\mathcal{C}_{\text {hor }}(R)$ denote the event that there exists an open path in $R$ from $\{a\} \times[c, d]$ to $\{b\} \times[c, d]$. For the probability of this event, we have a lower bound which is uniform in the size of the rectangle (but which depends on the shape, and is not expected to hold for $q=4$ ). 
Proposition 4.2 ([10], Theorem 7). Let $1 \leq q<4$ and $u>0$, and denote by $R_{n}^{u}$ the rectangle $[[0, u n]] \times[[0, n]]$ for $n \geq 1$. Then there exists a constant $\theta(u)>0$ such that

$$
\phi_{p_{c}(q), q, R_{n}^{u}}^{0}\left[\mathcal{C}_{\text {hor }}\left(R_{n}^{u}\right)\right] \geq \theta(u) \quad \text { for any } n \geq 1
$$

Next, we consider interfaces. If $(\mathcal{G} ; u, v)$ is a discrete Dobrushin domain, in the Dobrushin boundary conditions for the random-cluster model, all edges along the arc $(v u)$ are wired and all edges along $(u v)$ are free. Then, for each vertex $w$ of the medial graph $\mathcal{G}^{\diamond}$, there exists either an open edge of $\mathcal{G}$ or a dual-open edge of $\mathcal{G}^{*}$ passing through $w$. In addition, we can draw self-avoiding loops on $\mathcal{G}^{\diamond}$ as follows: a loop arriving at a vertex of the medial lattice always makes a turn of $\pm \pi / 2$, so as not to cross the open or dual-open edges through this vertex. The loop representation contains loops and the self-avoiding path connecting two vertices $u^{\diamond}$ and $v^{\diamond}$ of the medial graph $\mathcal{G}^{\diamond}$ that are closest to $u$ and $v$. This curve is called the interface (the exploration path) of the random-cluster model.

At the critical point $p=p_{c}(q)$, this interface is expected to converge weakly in the scaling limit to the chordal $\mathrm{SLE}_{\kappa}$ curve, with $\kappa$ specifically given by $q$. The convergence has been rigorously established for the special case of $q=2$, also known as the FK-Ising model [5], in the topology of Section 1.2.

Conjecture 4.3 (See, e.g., [35]). Let $0 \leq q \leq 4$ and $p=p_{c}(q)$. Let $\left(\Omega^{\delta} ; x^{\delta}, y^{\delta}\right)$ be a sequence of discrete Dobrushin domains converging to a Dobrushin domain $(\Omega ; x, y)$ in the Carathéodory sense. Then, as $\delta \rightarrow 0$, the interface of the critical random-cluster model on $\left(\Omega^{\delta} ; x^{\delta}, y^{\delta}\right)$, with cluster weight $q$ and Dobrushin boundary conditions, converges weakly to the chordal $\mathrm{SLE}_{\kappa}$ connecting $x$ and $y$ with

$$
\kappa=\frac{4 \pi}{\arccos (-\sqrt{q} / 2)} .
$$

THEOREM 4.4 ([5], Theorem 2). Conjecture 4.3 holds for $q=2$ and $\kappa=16 / 3$.

4.2. Existence of global multiple SLEs with $\kappa \in(4,6]$. We consider the convergence of random-cluster interfaces in the following setup. Abusing and lightening notation, let us write $x^{\delta}$ for both $x^{\delta}$ and $\left(x^{\diamond}\right)^{\delta}$ (converging to the same point $x$ as $\delta \rightarrow 0$ ). Let the polygons $\left(\Omega^{\delta} ; x_{1}^{\delta}, \ldots, x_{2 N}^{\delta}\right)$ converge to $\left(\Omega ; x_{1}, \ldots, x_{2 N}\right)$ as $\delta \rightarrow 0$ in the Carathéodory sense. Consider the critical random-cluster model on $\Omega^{\delta}$ with alternating boundary conditions (1.2). With such boundary conditions, there are $N$ interfaces $\left(\eta_{1}^{\delta}, \ldots, \eta_{N}^{\delta}\right)$ connecting pairwise the $2 N$ boundary points $x_{1}^{\delta}, \ldots, x_{2 N}^{\delta}$, as illustrated in Figure 5 . These interfaces form a planar connectivity encoded in a link pattern $\vartheta^{\delta} \in \mathrm{LP}_{N}$. We consider the interfaces conditionally on forming a given connectivity $\vartheta^{\delta}=\alpha=\left\{\left\{a_{1}, b_{1}\right\}, \ldots,\left\{a_{N}, b_{N}\right\}\right\} \in \mathrm{LP}_{N}$.

Conjecturally, conditionally on the event $\left\{\vartheta^{\delta}=\alpha\right\}$, the law of the collection $\left(\eta_{1}^{\delta}, \ldots, \eta_{N}^{\delta}\right)$ converges weakly as $\delta \rightarrow 0$ to a global $N-\mathrm{SLE}_{\kappa}$ associated to $\alpha$, where $\kappa$ is determined by $q$ via (4.2). In this section, we will prove this statement for the case of $q=2$ (so $\kappa=16 / 3$ ) this is the content of Proposition 1.4. The main inputs to the proof are Theorem 4.4 concerning convergence of one interface, classification of multiple SLE $16 / 3$ measures analogously to Theorem 1.2, and the RSW estimate from Proposition 4.2.

Proof of Proposition 1.4. Conditionally on $\left\{\vartheta^{\delta}=\alpha\right\}$, we have

$$
\left(\eta_{1}^{\delta}, \ldots, \eta_{N}^{\delta}\right) \in X_{0}^{\alpha}\left(\Omega^{\delta} ; x_{1}^{\delta}, \ldots, x_{2 N}^{\delta}\right) \quad \text { for all } \delta>0 .
$$




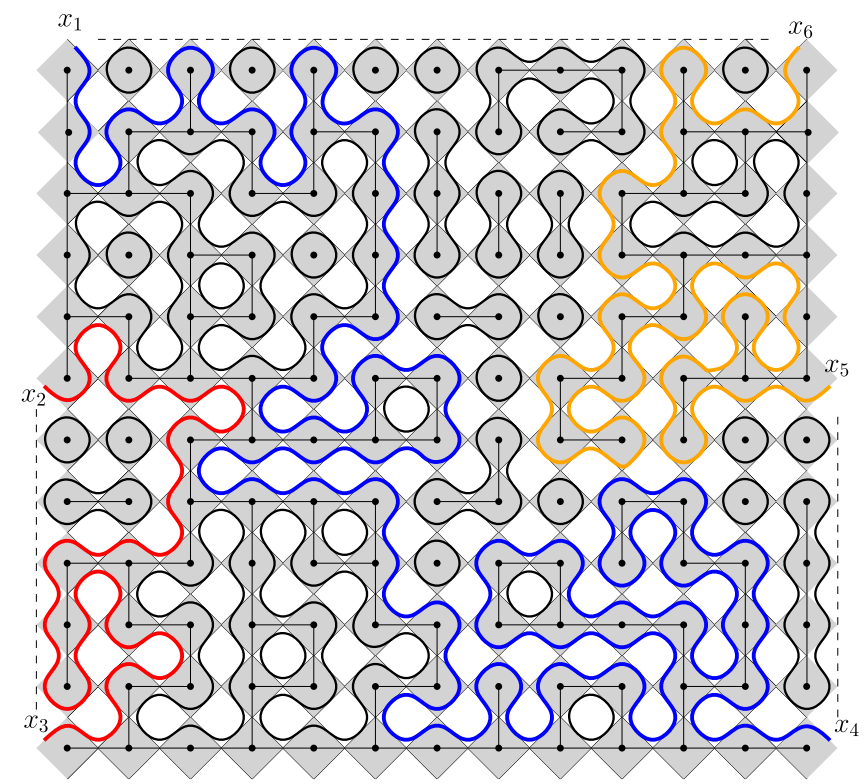

FIG. 5. The loop representation of a configuration of the random-cluster model on a polygon with six marked points $x_{1}, \ldots, x_{6}$ on the boundary, with alternating boundary conditions. There are three interfaces connecting the marked boundary points, illustrated in red, blue and orange, respectively.

First of all, the collection of laws of the sequence $\left(\left(\eta_{1}^{\delta}, \ldots, \eta_{N}^{\delta}\right)\right)_{\delta>0}$ is relatively compact; indeed, the RSW estimate in Proposition 4.2 implies the relative compactness by the results in $[1,19]$ (see Lemma 4.5 below). Thus, there exist subsequential limits, and we may assume that, for some sequence $\delta_{n} \stackrel{n \rightarrow \infty}{\longrightarrow} 0$, the sequence $\left(\eta_{1}^{\delta_{n}}, \ldots, \eta_{N}^{\delta_{n}}\right)$ converges weakly to $\left(\eta_{1}, \ldots, \eta_{N}\right)$. For convenience, we couple them in the same probability space so that they converge almost surely. Also, to lighten notation, we replace the superscripts $\delta_{n}$ by the superscript $n$ here and in what follows. For each $j \in\{1, \ldots, N\}$, we let $D_{j}^{n}$ denote the connected component of $\Omega^{n} \backslash \bigcup_{i \neq j} \eta_{i}^{n}$ having $x_{a_{j}}^{n}$ and $x_{b_{j}}^{n}$ on its boundary (see Figure 6 (left)).

In Lemma 4.6, we show that as $n \rightarrow \infty$, the discrete Dobrushin domains $\left(D_{j}^{n} ; x_{a_{j}}^{n}, x_{b_{j}}^{n}\right)$ converge almost surely to some random Dobrushin domains in the Carathéodory sense. Notice that it is not clear a priori that the limit of $D_{j}^{n}$ is still simply connected, as the interfaces in
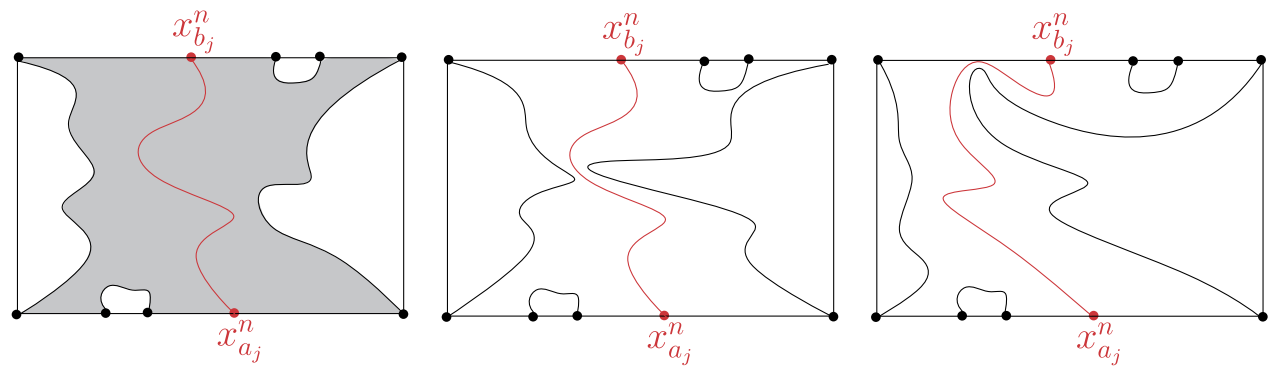

FIG. 6. In the left panel, the red curve is the interface connecting $x_{a_{j}}$ and $x_{b_{j}}$, and the gray part is $D_{j}^{n}$. The middle panel depicts a bulk pinching scenario, where around the bulk pinching point, an interior six-arm event (with alternating pattern) occurs. The right panel depicts a boundary pinching scenario, where around the boundary pinching point, a near-boundary three-arm event (with alternating pattern) occurs. We show in the proof of Lemma 4.6 that these events will not survive in the scaling limit. It turns out that in our case, the RSW Proposition 4.2 is sufficient for this purpose; note, however, that usually such bulk pinching events are ruled out by a six-arm exponent argument. 
the limit may touch the boundary, and they may have multiple points. The task in the proof of Lemma 4.6 is therefore to rule out this behavior. We establish this by using the RSW estimate from Proposition 4.2. Specifically, we show that the limit domain $\left(D_{j} ; x_{a_{j}}, x_{b_{j}}\right)$ is the simply connected subdomain $D_{j}$ of $\Omega \backslash \bigcup_{i \neq j} \eta_{i}$ with $x_{a_{j}}$ and $x_{b_{j}}$ on its boundary. As a by-product, Lemma 4.6 also shows that $\left(\eta_{1}, \ldots, \eta_{N}\right) \in X_{0}^{\alpha}\left(\Omega ; x_{1}, \ldots, x_{2 N}\right)$ almost surely.

Then, in Lemma 4.7 we prove that the subsequential limit $\left(\eta_{1}, \ldots, \eta_{N}\right)$ must be a global multiple $\mathrm{SLE}_{16 / 3}$. This also shows that global multiple $\mathrm{SLE}_{16 / 3}$ exists. Finally, in Proposition 4.10 in Section 4.3, we prove that such a global multiple SLE $_{16 / 3}$ is unique, thus being the unique subsequential limit. This gives the convergence of the sequence and concludes the proof of Proposition 1.4.

Next, we prove the auxiliary results needed to finish the proof of Proposition 1.4. We formulate some of them for general $q \in[1,4)$ (i.e., $\kappa \in(4,6]$ ), given Conjecture 4.3 , and discuss in Remark 4.12 the scope of these results.

LEMMA 4.5. Suppose Conjecture 4.3 holds for some $q \in[1,4)$ and let $\kappa \in(4,6]$ be the value related to $q$ via (4.2). Then the collection of laws of the sequence $\left(\left(\eta_{1}^{\delta}, \ldots, \eta_{N}^{\delta}\right)\right)_{\delta>0}$ is relatively compact.

PROOF. The RSW estimate in Proposition 4.2 shows that the single FK-Ising interface with Dobrushin boundary conditions satisfies the so-called condition "G2" in [19], and thus, its law is relatively compact, as stated in [10], Theorem 6 . This can be generalized to conclude that also the sequence $\left(\left(\eta_{1}^{\delta}, \ldots, \eta_{N}^{\delta}\right)\right)_{\delta>0}$ of multiple interfaces with alternating boundary conditions is relatively compact; see [17], Theorem 4.1, for details.

LEMMA 4.6. Suppose Conjecture 4.3 holds for some $q \in[1,4)$ and let $\kappa \in(4,6]$ be the value related to $q$ via (4.2). As $n \rightarrow \infty$, for each $j \in\{1, \ldots, N\}$, the discrete Dobrushin domain $\left(D_{j}^{n} ; x_{a_{j}}^{n}, x_{b_{j}}^{n}\right)$ converges almost surely to the Dobrushin domain $\left(D_{j} ; x_{a_{j}}, x_{b_{j}}\right)$ in the Carathéodory sense.

Proof. Fix $j \in\{1, \ldots, N\}$. As $n \rightarrow \infty$, the domains $\left(D_{j}^{n} ; x_{a_{j}}^{n}, x_{b_{j}}^{n}\right)$ can fail to converge to a Dobrushin domain only if the limit domain $D_{j}$ is not simply connected. There are two scenarios when this could happen, both resulting from specific behavior of the other interfaces $\eta_{i}^{n}$ with $i \neq j$ : either two of these interfaces get close together in the interior of $\Omega^{n}$, pinching $\eta_{j}^{n}$ in between (see Figure 6 (middle)), or one of these interfaces gets close to the boundary of $\Omega^{n}$, pinching $\eta_{j}^{n}$ to the boundary (see Figure 6(right)). In both cases, the points $x_{a_{j}}^{n}$ and $x_{b_{j}}^{n}$ get disconnected in the limit $n \rightarrow \infty$. We call the former a bulk pinching scenario and the latter a boundary pinching scenario.

First, we consider the boundary pinching scenario. Without loss of generality, we may assume that the boundary conditions on $\left(x_{a_{j}}^{n} x_{a_{j}+1}^{n}\right)$ are wired, as in Figure 7 (left). Also, it suffices to consider the pinching on the boundary arc $\left(x_{b_{j}}^{n} x_{b_{j}+1}^{n}\right)$ and assume that $b_{j} \geq a_{j}+2$. Denote by $\mathcal{C}_{j}^{n}$ the event that there is an open path connecting $\left(x_{a_{j}}^{n} x_{a_{j}+1}^{n}\right)$ to $\left(x_{b_{j}-1}^{n} x_{b_{j}}^{n}\right)$ in $\Omega^{n}$. Note that $\left\{\vartheta^{n}=\alpha\right\}$ implies the event $\mathcal{C}_{j}^{n}$. Denote the exploration path from $x_{a_{j}+1}^{n}$ to $x_{b_{j}-1}^{n}$ by $\zeta_{j}^{n}$, as in Figure 7 (middle), parameterized by the number of steps starting from $x_{a_{j}+1}^{n}$. For a fixed time $t$, inside the domain $\Omega^{n} \backslash \zeta_{j}^{n}[0, t]$, consider the two boundary $\operatorname{arcs} \partial_{1}^{n}:=\left(x_{b_{j}}^{n} x_{b_{j}+1}^{n}\right)$ and $\partial_{2}^{n}$ defined as the union of the boundary arc $\left(x_{a_{j}+1}^{n} x_{a_{j}+2}^{n}\right)$ and the right-hand side of $\zeta_{j}^{n}[0, t]$ - both carry free boundary conditions, and are drawn in red on Figure 7(right). Notice that the event $\mathcal{C}_{j}^{n}$ implies that there is no dual-open crosscut between $\partial_{1}^{n}$ and $\partial_{2}^{n}$. The gist 


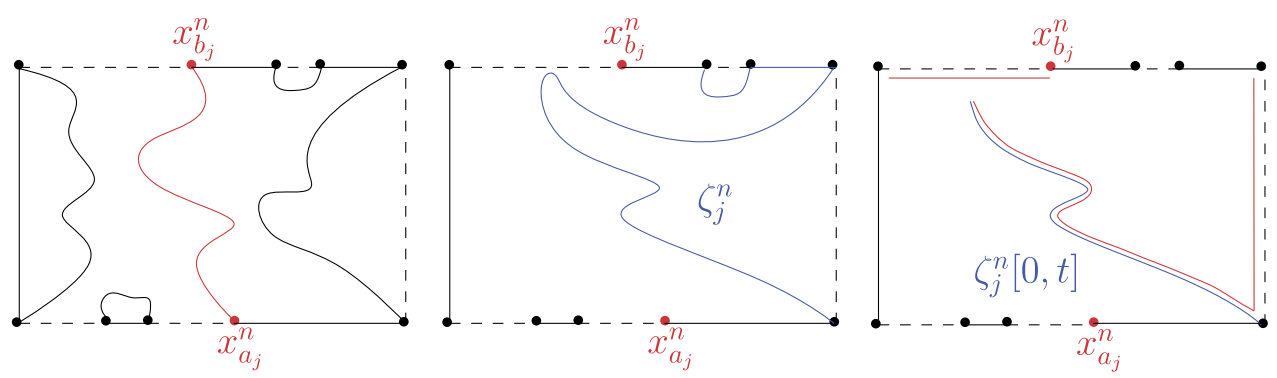

FIG. 7. A typical boundary pinching scenario.

of the argument is that if a boundary pinching occurs, such a crosscut will exist with high probability.

For all $t \geq 0$, let $d_{1}^{n}(t)$ denote the length of the shortest path in $D_{j}^{n}$ between $\zeta_{j}^{n}(t)$ and $\partial_{1}^{n}$ that does not intersect $\zeta_{j}^{n}[0, t]$, and set $d_{2}^{n}(t):=\left|x_{b_{j}}^{n}-\zeta_{j}^{n}(t)\right|$ and $\varepsilon_{j}^{n}(t):=d_{1}^{n}(t) / d_{2}^{n}(t)$. Then the RSW estimate from Proposition 4.2 combined with the FKG inequality (4.1) shows that for some universal constant $C>0$, we have the upper bound $\mathbb{P}\left[\mathcal{C}_{j}^{n} \mid \zeta_{j}^{n}[0, t]\right] \leq C\left(\varepsilon_{j}^{n}(t)\right)^{1 / C}$. Now, for $u>0$ small, denote by $T_{u}$ the first time $t \geq 0$ when $\varepsilon_{j}^{n}(t) \leq u$ (equaling $+\infty$ if no such time exists). From the above bound, we obtain

$$
\begin{aligned}
\mathbb{P}\left[\inf _{t \geq 0} \varepsilon_{j}^{n}(t) \leq u \mid \vartheta^{n}=\alpha\right] & \leq \frac{\mathbb{P}\left[\mathcal{C}_{j}^{n} \cap\left\{\inf _{t \geq 0} \varepsilon_{j}^{n}(t) \leq u\right\}\right]}{\mathbb{P}\left[\vartheta^{n}=\alpha\right]} \\
& =\frac{\mathbb{E}\left[\mathbb{1}_{\left\{T_{u}<\infty\right\}} \mathbb{E}\left[\mathcal{C}_{j}^{n} \mid \zeta_{j}^{n}\left[0, T_{u}\right]\right]\right]}{\mathbb{P}\left[\vartheta^{n}=\alpha\right]} \\
& \leq \frac{C u^{1 / C}}{\mathbb{P}\left[\vartheta^{n}=\alpha\right]} .
\end{aligned}
$$

Proposition 4.2 (cf. the footnote in the proof of Lemma 4.11) implies that $\mathbb{P}\left[\vartheta^{n}=\alpha\right]$ is bounded away from zero uniformly in $n$. Therefore, we have

$$
\lim _{u \rightarrow 0} \limsup _{n \rightarrow \infty} \mathbb{P}\left[\inf _{t \geq 0} \varepsilon_{j}^{n}(t) \leq u \mid \vartheta^{n}=\alpha\right]=0 .
$$

This shows that, in the scaling limit $n \rightarrow \infty$, the boundary pinching scenario cannot occur.

Bulk pinchings can be ruled out as a consequence. Indeed, assume that on the boundary of the domain $\Omega^{n}$, there is a triple of pairs of boundary points belonging to the pairing $\vartheta^{n}$ and such that the corresponding interfaces, say $\zeta_{1}^{n}, \zeta_{2}^{n}$ and $\zeta_{3}^{n}$, are involved in a bulk pinching scenario with positive probability (see Figure 6 (middle), where $\zeta_{2}^{n}$ is colored red). First, explore $\zeta_{1}^{n}$; such a bulk pinching can then be seen as a boundary pinching in the complement of $\zeta_{1}^{n}$, and such boundary pinchings are excluded by the previous argument.

In summary, we have shown that neither the bulk pinching scenario nor the boundary pinching scenario can survive in the scaling limit. This shows that $\left(D_{j}^{n} ; x_{a_{j}}^{n}, x_{b_{j}}^{n}\right)$ converges almost surely to the Dobrushin domain $\left(D_{j} ; x_{a_{j}}, x_{b_{j}}\right)$ in the Carathéodory sense, which is what we sought to prove.

Note that the proof of Lemma 4.6 also shows that almost surely,

$$
\left(\eta_{1}, \ldots, \eta_{N}\right) \in X_{0}^{\alpha}\left(\Omega ; x_{1}, \ldots, x_{2 N}\right)
$$

LEMMA 4.7. In the setup of the proof of Proposition 1.4 (with $q=2$ and $\kappa=16 / 3$ ), the limit $\left(\eta_{1}, \ldots, \eta_{N}\right)$ has the distribution of a global multiple $\mathrm{SLE}_{16 / 3}$. 
ProOF. We need to prove that, for each $j \in\{1, \ldots, N\}$, the conditional law of the random curve $X:=\eta_{j}$ given the other random curves $Y:=\left(\eta_{1}, \ldots, \eta_{j-1}, \eta_{j+1}, \ldots, \eta_{N}\right)$ is the appropriate chordal $\mathrm{SLE}_{16 / 3}$. We fix $j$ and denote

$$
X^{n}:=\eta_{j}^{n} \quad \text { and } \quad Y^{n}:=\left(\eta_{1}^{n}, \ldots, \eta_{j-1}^{n}, \eta_{j+1}^{n}, \ldots, \eta_{N}^{n}\right), \quad n \geq 1 .
$$

By assumption, $\left(X^{n}, Y^{n}\right)$ converges to $(X, Y)$ in distribution. However, this does not automatically imply the convergence of the conditional distribution of $X^{n}$ given $Y^{n}$ to the conditional distribution of $X$ given $Y$. In our case this is true, as we will now prove. (See also the discussion in [12], Section 5.)

Recall that we couple all of the random variables $\left\{\left(X^{n}, Y^{n}\right): n \geq 1\right\}$ in the same probability space so that they converge almost surely to $(X, Y)$ as $n \rightarrow \infty$. Now, given $Y^{n}$, the random curve $X^{n}$ is a FK-Ising interface with Dobrushin boundary conditions in the random Dobrushin domain $\left(D_{j}^{n} ; x_{a_{j}}^{n}, x_{b_{j}}^{n}\right)$ by the domain Markov property. By Lemma 4.6, $\left(D_{j}^{n} ; x_{a_{j}}^{n}, x_{b_{j}}^{n}\right)$ converges almost surely to the random Dobrushin domain $\left(D_{j} ; x_{a_{j}}, x_{b_{j}}\right)$ in the Carathéodory sense. Thus, almost surely, there exist conformal maps $G^{n}$ (resp., $G$ ) from $\mathbb{U}$ onto $D_{j}^{n}$ (resp., $D_{j}$ ) such that, as $n \rightarrow \infty$, the maps $G^{n}$ converge to $G$ uniformly on compact subsets of $\mathbb{U}$, and we have $\left(G^{n}\right)^{-1}\left(x_{a_{j}}^{n}\right) \rightarrow G^{-1}\left(x_{a_{j}}\right)=1$ and $\left(G^{n}\right)^{-1}\left(x_{b_{j}}^{n}\right) \rightarrow G^{-1}\left(x_{b_{j}}\right)=$ -1 . Furthermore, for each $n$, the map $G^{n}$ is a measurable function of $Y^{n}$, and $G$ is a measurable function of $Y$. To conclude, we use the following two observations:

1. On the one hand, Theorem 4.4 shows that the law of $\left(G^{n}\right)^{-1}\left(X^{n}\right)$ converges to the chordal $\mathrm{SLE}_{3}$ in $\mathbb{U}$ connecting the points 1 and -1 .

2. On the other hand (see also [16], Proposition 4.7), one can show that $\left(G^{n}\right)^{-1}\left(X^{n}\right)$ converges to $G^{-1}(X)$ as follows. By assumption, $\left(X^{n}, Y^{n}\right)$ converges to $(X, Y)$ almost surely. Now, we send $X^{n}$ (resp., $X$ ) conformally onto $\mathbb{H}$ and denote by $W^{n}$ (resp., $W$ ) its driving function. On the one hand, applying [19], Proposition 4.12, Theorem 1.5, and Corollary 1.7 , to the critical FK-Ising interfaces $\left(X^{n}\right)_{n \geq 1}$, we see that $W^{n} \rightarrow W$ locally uniformly. On the other hand, applying [19], Proposition 4.12, Theorem 1.5 and Corollary 1.7, to $\left\{\left(G^{n}\right)^{-1}\left(X^{n}\right)\right\}$, we see that this collection is tight, and for any convergent subsequence $\left(G^{n_{k}}\right)^{-1}\left(X^{n_{k}}\right) \rightarrow \tilde{\eta}$, the curve $\tilde{\eta}$ has a continuous driving function $\widetilde{W}$ such that $W^{n_{k}} \rightarrow \widetilde{W}$ locally uniformly (note that this fact is highly nontrivial). Combining these two facts, we see that $\widetilde{W}$ coincides with $W$, so $\tilde{\eta}$ coincides with $G^{-1}(X)$. In particular, this is the only subsequential limit of the collection $\left\{\left(G^{n}\right)^{-1}\left(X^{n}\right): n \geq 1\right\}$, so we have $\left(G^{n}\right)^{-1}\left(X^{n}\right) \rightarrow G^{-1}(X)$ as $n \rightarrow \infty$.

Combining these observations, we see that the law of $G^{-1}(X)$ is the chordal $\mathrm{SLE}_{16 / 3}$ in $\mathbb{U}$ connecting 1 and -1 . In particular, the law of $G^{-1}(X)$ is independent of $Y$ with $G$ a measurable function of $Y$. Hence, the conditional law of $X$ given $Y$ is the chordal $\mathrm{SLE}_{16 / 3}$ in $D_{j}$ connecting the points $x_{a_{j}}$ and $x_{b_{j}}$.

4.3. Uniqueness of global multiple SLEs with $\kappa \in(4,6]$. In this section, we prove that the scaling limit of each subsequence of FK-Ising interfaces is unique, thereby completing the proof of Proposition 1.4. The idea is similar to the proof of Theorem 1.2 in Section 3.3. In particular, we need analogues of the lemmas appearing in Sections 3.2 and 3.3. Again, we formulate them for general $\kappa \in(4,6]$.

LEMMA 4.8. Suppose Conjecture 4.3 holds for some $q \in[1,4)$ and let $\kappa \in(4,6]$ be the value related to $q$ via (4.2). Let $(\Omega ; x, y)$ be a bounded Dobrushin domain. Let $\Omega^{L}, U \subset \Omega$ be Dobrushin subdomains such that $\Omega^{L}, U$, and $\Omega$ agree in a neighborhood of the arc $(y x)$. Suppose $\gamma \sim \mathbb{P}(\Omega ; x, y)$ and $\eta \sim \mathbb{P}(U ; x, y)$. Then we have

$$
\mathbb{P}\left[\eta \subset \Omega^{L}\right] \geq \mathbb{P}\left[\gamma \subset \Omega^{L}\right] .
$$

In particular, Lemma 3.5 holds for the corresponding $\kappa \in(4,6]$. 
PROOF. This immediately follows by combining the domain Markov property with the comparison (4.1) of boundary conditions with Conjecture 4.3.

We remark that Lemma 4.8 concerns the chordal $\operatorname{SLE}_{\kappa}$ with $\kappa \in(4,6]$, and its statement has nothing to do with discrete models. However, we do not have a proof for this lemma without using Conjecture 4.3.

Proposition 4.9. Suppose Conjecture 4.3 holds for some $q \in[1,4)$ and let $\kappa \in(4,6]$ be the value related to $q$ via (4.2). Then, for each quad $\left(\Omega ; x_{1}, \ldots, x_{4}\right)$ and for each link pattern $\alpha \in \mathrm{LP}_{2}$, there exists a unique global 2-SLE $\mathrm{SL}_{\kappa}$ associated to $\alpha$.

Proof. As in Section 3.2, without loss of generality, we assume that $\alpha=\{\{1,4\},\{2,3\}\}$. Then, to prove the assertion, we argue as in the proof of Proposition 3.2, with $\left(\Omega ; x_{1}, \ldots\right.$, $\left.x_{4}\right)=\left(\Omega ; x^{L}, x^{R}, y^{R}, y^{L}\right)$. Taking $\Omega=[0, \ell] \times[0,1]$ and $x^{L}=(0,0), x^{R}=(\ell, 0), y^{R}=$ $(\ell, 1), y^{L}=(0,1)$, we define a Markov chain on pairs $\left(\eta^{L}, \eta^{R}\right)$ of curves by sampling from the conditional laws: given $\left(\eta_{n}^{L}, \eta_{n}^{R}\right)$, we pick $i \in\{L, R\}$ uniformly and resample $\eta_{n+1}^{i}$ according to the conditional law given the other curve. However, in the current situation, we have $\kappa \in(4,6]$, so the configuration sampled according to this rule may no longer stay in the space $X_{0}\left(\Omega ; x^{L}, y^{L}, x^{R}, y^{L}\right)$. In this case, when resampling according to the conditional law, we sample the curves in each connected component and concatenate the pieces of curves together; see the more detailed description beneath equation (4.4). Fortunately, this issue turns out to be irrelevant in the end, as we will show that, for any initial configuration $\left(\eta_{0}^{L}, \eta_{0}^{R}\right) \in X_{0}\left(\Omega ; x^{L}, x^{R}, y^{R}, y^{L}\right)$, the corresponding Markov chain $\left(\eta_{n}^{L}, \eta_{n}^{R}\right)$ will eventually stay in the space $X_{0}\left(\Omega ; x^{L}, y^{L}, x^{R}, y^{L}\right)$ :

$$
\mathbb{P}\left[\exists n_{0}<\infty \text { such that }\left(\eta_{n}^{L}, \eta_{n}^{R}\right) \in X_{0}\left(\Omega ; x^{L}, y^{L}, x^{R}, y^{L}\right) \text { for all } n \geq n_{0}\right]=1 .
$$

Once (4.3) has been established, the existence and uniqueness of the global 2-SLE follows by repeating the proof Proposition 3.2, with Lemma 3.5 replaced by Lemma 4.8. Hence, it remains to prove (4.3).

In the Markov chain $\left(\eta_{n}^{L}, \eta_{n}^{R}\right)$, we want to record the times when $L$ and $R$ are picked. Let $\tau_{0}^{L}=\tau_{0}^{R}=0$, and for $n \geq 1$, let $\tau_{n}^{R}$ (resp., $\tau_{n}^{L}$ ) be the first time after $\tau_{n-1}^{L}$ (resp., $\tau_{n}^{R}$ ) that $R$ (resp., $L$ ) is picked. Let

$$
n_{\kappa}=\left\lceil\frac{\kappa}{8-\kappa}\right\rceil+1 \text {. }
$$

To prove (4.3), it suffices to show that $\eta_{n}^{R} \cap\left(y^{L} x^{L}\right)=\varnothing$ for all $n \geq \tau_{n_{\kappa}}^{R}$, because a similar property for $\eta_{n}^{L}$ follows by symmetry (note also that $\tau_{n}^{L} \geq \tau_{n}^{R}$ ). For this purpose, we let $\gamma^{R}$ be the $\mathrm{SLE}_{\kappa}$ in $\Omega$ connecting $x^{R}$ and $y^{R}$. We will use the following two essential properties of $\gamma^{R}$ :

1. By the duality property of the $\mathrm{SLE}_{\kappa}$ (see, e.g., [9] or [29], Theorem 1.4), we know that the left boundary of $\gamma^{R}$ has the law of the $\operatorname{SLE}_{16 / \kappa}(16 / \kappa-4 ; 8 / \kappa-2)$ with two force points next to the starting point. Therefore, the left boundary of $\gamma^{R}$ does not hit $\left(x^{R} y^{R}\right)$.

2. The curve $\gamma^{R}$ hits $\left(y^{L} x^{L}\right)$ with positive probability, and using [2] and Lemma A.1 from the Appendix, we see that, almost surely on the event $\left\{\gamma^{R} \cap\left(y^{L} x^{L}\right) \neq \varnothing\right\}$, the Hausdorff dimension of the intersection set satisfies

$$
\operatorname{dim}\left(\gamma^{R} \cap\left(y^{L} x^{L}\right)\right) \leq 1-\beta, \quad \text { where } \beta=\frac{8-\kappa}{\kappa} .
$$


Now, for $\tau_{1}^{R} \leq n \leq \tau_{1}^{L}-1$, the curve $\eta_{n}^{R}$ is an $\mathrm{SLE}_{\kappa}$ in a domain which is a subset of $\Omega$. By Lemma 4.8, we can couple $\eta_{n}^{R}$ and $\gamma^{R}$ so that $\gamma^{R}$ stays to the left of $\eta_{n}^{R}$ almost surely. Thus, we have almost surely

$$
\operatorname{dim}\left(\eta_{n}^{R} \cap\left(y^{L} x^{L}\right)\right) \leq \operatorname{dim}\left(\gamma^{R} \cap\left(y^{L} x^{L}\right)\right) \leq 1-\beta .
$$

In particular, for the last time before sampling the left curve, we have almost surely

$$
\operatorname{dim}\left(A_{1}\right) \leq 1-\beta \quad \text { for } A_{1}=\eta_{\tau_{1}^{L}-1}^{R} \cap\left(y^{L} x^{L}\right) .
$$

Then, for $\tau_{1}^{L} \leq n \leq \tau_{2}^{R}-1$, we sample $\eta_{n}^{L}$ according to the conditional law given $\eta_{\tau_{1}^{L}-1}^{R}$. However, if $A_{1} \neq \varnothing$, then the domain $\Omega \backslash \eta_{\tau^{L}-1}^{R}$ is not connected. In this case, we sample the $\mathrm{SLE}_{\kappa}$ in those connected components of $\tau_{S}^{L} \backslash \eta_{\tau^{L}-1}^{R}$ which have a part of $\left(y^{L} x^{L}\right)$ on the boundary and define $\eta_{n}^{L}$ to be the concatenation of these curves. We note that, by the above observation 1 , the right boundary of $\eta_{n}^{L}$ only hits $\left(y^{L} x^{L}\right)$ in $A_{1}$.

Next, for $\tau_{2}^{R} \leq n \leq \tau_{2}^{L}-1$, we sample $\eta_{n}^{R}$ according to the conditional law given $\eta_{\tau_{2}^{R}-1}^{L}$. Again, the curve $\eta_{n}^{R}$ is a SLE in $_{\kappa}$ a domain which is a subset of $\Omega$, and we can couple it with $\gamma^{R}$ in such a way that $\gamma^{R}$ stays to the left of $\eta_{n}^{R}$ almost surely. Thus, we have almost surely

$$
\eta_{n}^{R} \cap\left(y^{L} x^{L}\right) \subset \eta_{n}^{R} \cap A_{1} \subset \gamma^{R} \cap A_{1} .
$$

Combining this with (4.4), we see that, almost surely,

$$
\operatorname{dim}\left(\eta_{n}^{R} \cap\left(y^{L} x^{L}\right)\right) \leq \operatorname{dim}\left(\gamma^{R} \cap A_{1}\right) \leq(1-2 \beta)^{+} .
$$

In particular, we can improve (4.4) to

$$
\operatorname{dim}\left(A_{2}\right) \leq(1-2 \beta)^{+} \text {for } A_{2}=\eta_{\tau_{2}^{L}-1}^{R} \cap\left(y^{L} x^{L}\right)
$$

and iterating the same argument and combining with Lemma A.1, we see that

$$
\eta_{n}^{R} \cap\left(y^{L} x^{L}\right)=\varnothing \quad \text { for all } n \geq \tau_{n_{\kappa}}^{R},
$$

almost surely. This concludes the proof.

Proposition 4.10. Let $\left(\Omega ; x_{1}, \ldots, x_{2 N}\right)$ be a polygon with $N \geq 1$. For any $\alpha \in \operatorname{LP}_{N}$, there exists a unique global $N-\mathrm{SLE}_{16 / 3}$ associated to $\alpha$.

ProOF. The existence follows from the subsequential scaling limit in Lemma 4.7, so it remains to prove the uniqueness. We use induction on $N \geq 2$ and the same arguments as in the proof of Theorem 1.2. First, the assertion holds for $N=2$ by Proposition 4.9. Next, we let $N \geq 3$ and assume that for any $\beta \in \mathrm{LP}_{N-1}$, the global $(N-1)-\mathrm{SLE}_{\kappa}$ associated to $\beta$ is unique. Then, as in the proof of Theorem 1.2, we take $\alpha \in \operatorname{LP}_{N}$ with $\{1,2\} \in \alpha$ and $\{r, r+1\} \in \alpha$ for some $r \in\{3,4, \ldots, 2 N-1\}$, and we let $\left(\eta_{1}, \ldots, \eta_{N}\right) \in X_{0}^{\alpha}\left(\Omega ; x_{1}, \ldots, x_{2 N}\right)$ be a global $N$-SLE ${ }_{\kappa}$ associated to $\alpha$. We denote by $\eta^{L}$ (resp., $\eta^{R}$ ) the curve in the collection $\left\{\eta_{1}, \ldots, \eta_{N}\right\}$ that connects $x_{1}$ and $x_{2}$ (resp., $x_{r}$ and $x_{r+1}$ ). By the induction hypothesis, given $\eta^{R}$ (resp., $\eta^{L}$ ), the conditional law of the rest of the curves is the unique global $(N-1)-\mathrm{SLE}_{\kappa}$ associated to $\alpha /\{r, r+1\}$ (resp., $\alpha /\{1,2\}$ ). This gives the conditional law of $\eta^{L}$ given $\eta^{R}$ and vice versa. One can then use the argument from the proof of Proposition 3.2, considering Markov chains sampling $\eta^{L}$ and $\eta^{R}$ from their conditional laws-one only has to replace Lemma 3.5 by Lemma 4.8 and Lemma 3.6 by the following Lemma 4.11 for $N-1$.

The next technical lemma can be thought of as an analogue of Lemma 3.6. To state it, we fix $\alpha \in \operatorname{LP}_{N}$ such that $\{1,2\} \in \alpha$ and let $\left(\Omega ; x_{1}, \ldots, x_{2 N}\right)$ be a bounded polygon. Also, if $\left(\eta_{1}, \ldots, \eta_{N}\right)$ is a family of random curves with the law of a global $N-\mathrm{SLE}_{\kappa}$ associated to $\alpha$, and if $\eta:=\eta_{1}$ is the curve connecting $x_{1}$ and $x_{2}$, then we denote by $\mathbb{Q}_{\alpha}^{\{1,2\}}\left(\Omega ; x_{1}, \ldots, x_{2 N}\right)$ the law of $\eta$. 
LEMMA 4.11. Assume that there exists a unique global $N-\mathrm{SLE}_{16 / 3}$ associated to $\alpha$. Let $\Omega^{L} \subset U, \tilde{U} \subset \Omega$ be subpolygons such that $\Omega^{L}$ and $\Omega$ agree in a neighborhood of the boundary arc $\left(x_{1} x_{2}\right)$. Suppose that $\eta \sim \mathbb{Q}_{\alpha}^{\{1,2\}}\left(U ; x_{1}, \ldots, x_{2 N}\right)$ and $\tilde{\eta} \sim \mathbb{Q}_{\alpha}^{\{1,2\}}\left(\tilde{U} ; x_{1}, \ldots, x_{2 N}\right)$. Then there exists a coupling $(\eta, \tilde{\eta})$ such that $\mathbb{P}\left[\eta=\tilde{\eta} \subset \Omega^{L}\right] \geq \theta$, where the constant $\theta=\theta\left(\Omega, \Omega^{L}\right)>0$ is independent of $U$ and $\tilde{U}$.

PRoOF. Let $\left(\Omega^{\delta} ; x_{1}^{\delta}, \ldots, x_{2 N}^{\delta}\right)$ be discrete polygons converging to $\left(\Omega ; x_{1}, \ldots, x_{2 N}\right)$ in the Carathéodory sense, and denote by $U^{\delta}, \tilde{U}^{\delta}$, and $\left(\Omega^{L}\right)^{\delta}$ the corresponding approximations of $U, \tilde{U}$, and $\Omega^{L}$. Also, let $\left(\eta_{1}^{\delta}, \ldots, \eta_{N}^{\delta}\right)$ (resp., $\left(\tilde{\eta}_{1}^{\delta}, \ldots, \tilde{\eta}_{N}^{\delta}\right)$ ) be the collection of interfaces in the critical random-cluster model on $U^{\delta}$ (resp., $\tilde{U}^{\delta}$ ) with alternating boundary conditions (1.2), and let $\eta^{\delta}:=\eta_{1}^{\delta}$ and $\tilde{\eta}^{\delta}:=\tilde{\eta}_{1}^{\delta}$ be the curves connecting $x_{1}^{\delta}$ and $x_{2}^{\delta}$. By the assumptions, we know that, as $\delta \rightarrow 0$, the law of $\eta^{\delta}$ (resp., $\tilde{\eta}^{\delta}$ ) conditionally on $\left\{\vartheta^{\delta}=\alpha\right\}$ (resp., $\left\{\tilde{\vartheta}^{\delta}=\alpha\right\}$ ) converges to $\mathbb{Q}_{\alpha}^{\{1,2\}}\left(U ; x_{1}, \ldots, x_{2 N}\right)$ (resp., $\mathbb{Q}_{\alpha}^{\{1,2\}}\left(\tilde{U} ; x_{1}, \ldots, x_{2 N}\right)$ ). Thus, it is sufficient to show that there exists a coupling $\left(\eta^{\delta}, \tilde{\eta}^{\delta}\right)$ such that $\mathbb{P}\left[\eta^{\delta}=\tilde{\eta}^{\delta} \subset\left(\Omega^{L}\right)^{\delta}\right] \geq \theta$ for $\delta$ small enough, where the constant $\theta=\theta\left(\Omega, \Omega^{L}\right)>0$ is independent of $U$ and $\tilde{U}$.

Since $\Omega^{L}$ agrees with $\Omega$ in a neighborhood of $\left(x_{1} x_{2}\right)$, we can find boundary points $y_{1}$ and $y_{2}$ such that $y_{1}, x_{1}, x_{2}, y_{2}$ lie in counterclockwise order along $\partial \Omega$ and $\Omega^{L}$ agrees with $\Omega$ in a neighborhood of $\left(y_{1} y_{2}\right)$. Now, we have wired boundary conditions on the $\operatorname{arc}\left(x_{1}^{\delta} x_{2}^{\delta}\right)$ and free boundary conditions on the $\operatorname{arcs}\left(x_{2}^{\delta} x_{3}^{\delta}\right)$ and $\left(x_{2 N}^{\delta} x_{1}^{\delta}\right)$. Define $\mathcal{C}_{*}^{\delta}$ to be the event that there exists a dual-open path in $\left(\Omega^{L}\right)^{\delta}$ from $\left(x_{2}^{\delta} y_{2}^{\delta}\right)$ to $\left(y_{1}^{\delta} x_{1}^{\delta}\right)$. Then, by the domain Markov property, there exists a coupling of $\eta^{\delta}$ and $\tilde{\eta}^{\delta}$ such that the probability of $\left\{\eta^{\delta}=\tilde{\eta}^{\delta} \subset\left(\Omega^{L}\right)^{\delta}\right\}$ is bounded from below by the minimum of $\mathbb{P}\left[\mathcal{C}_{*}^{\delta}\right]$ and $\tilde{\mathbb{P}}\left[\mathcal{C}_{*}^{\delta}\right]$, where $\mathbb{P}$ and $\tilde{\mathbb{P}}$ denote the probability measures of the random-cluster models on $U^{\delta}$ and $\tilde{U}^{\delta}$ with alternating boundary conditions (1.2). Furthermore, as a consequence of Proposition 4.2, the domain Markov property, and the FKG inequality (4.1), we obtain $\mathbb{P}\left[\mathcal{C}_{*}^{\delta}\right] \geq \theta\left(\Omega, \Omega^{L}\right)>0$ (and likewise for $\left.\tilde{U}\right){ }^{3}$ In particular, the lower bound $\theta\left(\Omega, \Omega^{L}\right)$ is uniform over $U$ (resp., $\left.\tilde{U}\right)$ and $\delta$. By the convergence of $\eta^{\delta}$ and $\tilde{\eta}^{\delta}$, we obtain a coupling of $\eta$ and $\tilde{\eta}$ such that the probability of $\left\{\eta=\tilde{\eta} \subset \Omega^{L}\right\}$ is bounded from below by $\theta\left(\Omega, \Omega^{L}\right)$.

By the above, we have now completed the proof of Proposition 1.4 (with $q=2$ and $\kappa=$ $16 / 3$ ). We summarize the key ingredients in the proof in the following remark.

REMARK 4.12. The proof of Proposition 1.4 consists of Lemmas 4.5-4.11 and Propositions 4.9 and 4.10 .

- Lemmas 4.5, 4.6 and 4.7 require the RSW estimate from Proposition 4.2.

- Lemma 4.8 requires the convergence of a single interface, given by Conjecture 4.3.

- The proof of Proposition 4.9 uses Lemma 4.8. Assuming Lemma 4.8, this works for all $\kappa \in(4,8)$.

- Note also that the proof of Proposition 4.9 uses the duality of the $\mathrm{SLE}_{\kappa}$, which is known for all $\kappa \in(4,8)[9,29]$.

- The proofs of Proposition 4.10 and Lemma 4.11 use the convergence of the multiple FKIsing interfaces; thus they also require Lemmas 4.5-4.8 and Proposition 4.9 as an input.

\footnotetext{
${ }^{3}$ Note that, here we only need the RSW Proposition 4.2, because the lower bound $\theta$ is allowed to depend on the domains $\Omega, \Omega^{L}$. To derive $\theta\left(\Omega, \Omega^{L}\right)$ from Proposition 4.2, one can draw a zigzag path of rectangles so that the first one intersects the boundary arc $\left(y_{1}^{\delta} x_{1}^{\delta}\right)$, the last one intersects the boundary arc $\left(x_{2}^{\delta} y_{2}^{\delta}\right)$, and the middle ones are inside $\left(\Omega^{L}\right)^{\delta}$, and observe that dual crossings of all these rectangles give a dual crossing from $\left(y_{1}^{\delta} x_{1}^{\delta}\right)$ to $\left(x_{2}^{\delta} y_{2}^{\delta}\right)$.
} 
Overall, the proofs of these results require the RSW estimate from Proposition 4.2 and the convergence a single interface (Conjecture 4.3). Therefore, knowing Conjecture 4.3, the analogous conclusions to Proposition 1.4 would extend to other values of $\kappa$.

4.4. The Ising model. Let $\mathcal{G}$ be a finite subgraph of $\mathbb{Z}^{2}$. The Ising model on $\mathcal{G}$ with free boundary condition is a random assignment $\sigma \in\{\ominus, \oplus\}^{V(\mathcal{G})}$ of spins $\sigma_{v} \in\{\ominus, \oplus\}$, where $\sigma_{v}$ denotes the spin at the vertex $v \in V(\mathcal{G})$. The Hamiltonian is defined by

$$
H_{\mathcal{G}}^{\text {free }}(\sigma)=-\sum_{v \sim w} \sigma_{v} \sigma_{w}
$$

The probability measure of the Ising model is given by the Boltzmann measure with Hamiltonian $H_{\mathcal{G}}^{\text {free }}$ and inverse-temperature $\beta>0$ :

$$
\mu_{\beta, \mathcal{G}}^{\text {free }}[\sigma]=\frac{\exp \left(-\beta H_{\mathcal{G}}^{\text {free }}(\sigma)\right)}{Z_{\beta, \mathcal{G}}^{\text {free }}}, \quad \text { where } Z_{\beta, \mathcal{G}}^{\text {free }}=\sum_{\sigma} \exp \left(-\beta H_{\mathcal{G}}^{\text {free }}(\sigma)\right) .
$$

Also, for $\tau \in\{\ominus, \oplus\}^{\mathbb{Z}^{2}}$, we define the Ising model with boundary condition $\tau$ via the Hamiltonian

$$
H_{\mathcal{G}}^{\tau}(\sigma)=-\sum_{\substack{v \sim w,\langle v, w\rangle \cap \mathcal{G} \neq \varnothing}} \sigma_{v} \sigma_{w}, \quad \text { where } \sigma_{v}=\tau_{v} \text { for all } v \notin \mathcal{G}
$$

In particular, if $(\mathcal{G} ; v, w)$ is a discrete Dobrushin domain, we may consider the Ising model with the following Dobrushin boundary conditions (domain-wall boundary conditions): we set $\oplus$ along the arc $(v w)$, and $\ominus$ along the complementary arc $(w v)$. More generally, we will consider the alternating boundary conditions (1.1), where $\oplus$ and $\ominus$ alternate along the boundary as in Figure 1.

As in the case of the random-cluster model, we have the following useful domain Markov property. Let $\mathcal{G} \subset \mathcal{G}^{\prime}$ be two finite subgraphs of $\mathbb{Z}^{2}$. Fix $\tau \in\{\ominus, \oplus\}^{\mathbb{Z}^{2}}$ and $\beta>0$. Let $X$ be a random variable, which is measurable with respect to the status of the vertices in the smaller graph $\mathcal{G}$. Then we have

$$
\mu_{\beta, \mathcal{G}^{\prime}}^{\tau}\left[X \mid \sigma_{v}=\tau_{v} \text { for all } v \in \mathcal{G}^{\prime} \backslash \mathcal{G}\right]=\mu_{\beta, \mathcal{G}}^{\tau}[X]
$$

The planar Ising model exhibits an order-disorder phase transition at a certain critical temperature: above this temperature, the configurations are disordered and below it, one observes large clusters of equal spins. At criticality, the configurations show self-similar behavior, and indeed, the critical planar Ising model is conformally invariant in the scaling limit [5-7, 14, $39,40]$. On the square lattice, the critical value of $\beta$ is

$$
\beta_{c}=\frac{1}{2} \log (1+\sqrt{2}) .
$$

In Proposition 1.3, we consider the scaling limit of Ising interfaces at criticality. Let $\left(\Omega_{*}^{\delta} ; x_{*}^{\delta}, y_{*}^{\delta}\right)$ be discrete Dobrushin domains, $\delta>0$, and consider the critical Ising model on the duals $\left(\Omega_{*}^{\delta} ; x_{*}^{\delta}, y_{*}^{\delta}\right)$ with Dobrushin boundary conditions. Let $x_{\diamond}^{\delta}$ and $y_{\diamond}^{\delta}$ be vertices on the medial lattice $\Omega_{\diamond}^{\delta}$ nearest to $x_{*}^{\delta}$ and $y_{*}^{\delta}$. Then we define the Ising interface as follows. It starts from $x_{\diamond}^{\delta}$, traverses on the primal lattice $\Omega^{\delta}$, and turns at every vertex of $\Omega^{\delta}$ in such a way that it always has dual-vertices with spin $\oplus$ on its left and spin $\ominus$ on its right. If there is an indetermination when arriving at a vertex (this may happen on the square lattice), it turns left. See also Figure 8 for an illustration. This interface converges weakly as $\delta \rightarrow 0$ to the chordal $\mathrm{SLE}_{\kappa}$ with $\kappa=3$ (in the topology of Section 1.2). 


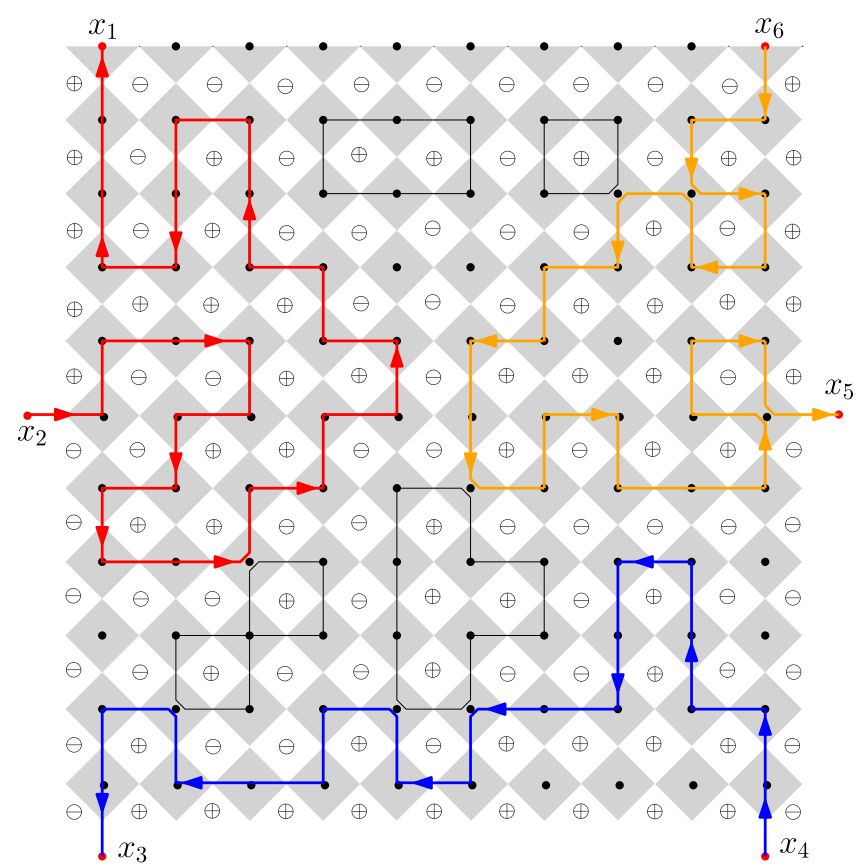

FIG. 8. A spin configuration of the Ising model on a polygon with six marked points $x_{1}, \ldots, x_{6}$ on the boundary, with alternating boundary conditions. There are three interfaces starting from $x_{2}, x_{4}$, and $x_{6}$, illustrated in red, blue and orange, respectively.

THEOREM 4.13 ([5], Theorem 1). Let $\left(\Omega_{*}^{\delta} ; x_{*}^{\delta}, y_{*}^{\delta}\right)$ be a sequence of discrete Dobrushin domains converging to a Dobrushin domain $(\Omega ; x, y)$ in the Carathéodory sense. Then, as $\delta \rightarrow 0$, the interface of the critical Ising model on $\left(\Omega_{*}^{\delta}, x_{*}^{\delta}, y_{*}^{\delta}\right)$ with Dobrushin boundary conditions converges weakly to the chordal $\mathrm{SLE}_{\kappa}$ in $\Omega$ connecting $x$ and $y$ with $\kappa=3$.

Using this result, we will prove that multiple interfaces also converge in the scaling limit to global multiple SLE 3 curves. Abusing and lightening notation, let us write $\Omega^{\delta}$ for $\Omega^{\delta},\left(\Omega^{\diamond}\right)^{\delta}$, or $\left(\Omega^{*}\right)^{\delta}$, and $x^{\delta}$ for $x^{\delta},\left(x^{\diamond}\right)^{\delta}$, or $\left(x^{*}\right)^{\delta}$. Let the polygons $\left(\Omega^{\delta} ; x_{1}^{\delta}, \ldots, x_{2 N}^{\delta}\right)$ converge to $\left(\Omega ; x_{1}, \ldots, x_{2 N}\right)$ as $\delta \rightarrow 0$ in the Carathéodory sense. Consider the critical Ising model on $\Omega^{\delta}$ with alternating boundary conditions (1.1). For $j \in\{1, \ldots, N\}$, let $\eta_{j}^{\delta}$ be the interface starting from $x_{2 j}^{\delta}$ that separates $\oplus$ from $\ominus$. Then the collection of interfaces $\left(\eta_{1}^{\delta}, \ldots, \eta_{N}^{\delta}\right)$ connects the boundary points $x_{1}^{\delta}, \ldots, x_{2 N}^{\delta}$ forming a planar link pattern $\vartheta^{\delta} \in \mathrm{LP}_{N}$. Proposition 1.3 asserts that conditionally on the event $\left\{\vartheta^{\delta}=\alpha\right\}$, the law of the collection $\left(\eta_{1}^{\delta}, \ldots, \eta_{N}^{\delta}\right)$ converges weakly as $\delta \rightarrow 0$ to a global $N-\mathrm{SLE}_{3}$ associated to $\alpha$. The proof of this is very similar to that of the FK-Ising model (Proposition 1.4)—we summarize it below.

Proof of Proposition 1.3. The uniqueness of the limit follows from Theorem 1.2 (the global $N-\mathrm{SLE}_{3}$ is unique). For the subsequential convergence, we follow the same lines as in the proof of Proposition 1.4. Recall the summary of its proof from Remark 4.12. First, for Lemmas 4.5 and 4.6, we need a RSW type estimate for the critical Ising model. This can be obtained from Proposition 4.2 via the so-called Edwards-Sokal coupling, as explained in [5], Remark 4. Then the proof of Lemma 4.7 holds for the critical Ising model and $\kappa=3$. Therefore, we conclude that for any convergent subsequence of $\left(\eta_{1}^{\delta_{n}}, \ldots, \eta_{N}^{\delta_{n}}\right)_{\delta_{n}>0}$, the limit must be a global multiple $N-\mathrm{SLE}_{3}$. Since the global $N-\mathrm{SLE}_{3}$ is unique due to Theorem 1.2, we readily establish the convergence of the whole sequence to this global $N-\mathrm{SLE}_{3}$. Finally, the asserted marginal law of $\eta_{j}$ follows from Lemma 3.12. 


\section{APPENDIX: INTERSECTION OF TWO FRACTALS}

For use in Section 4, we record in this Appendix some properties of random subsets of the boundary of the unit disc $\mathbb{U}=\{z \in \mathbb{C}:|z| \leq 1\}$. In spite of stating the results for $\mathbb{U}$, we may as well apply the following lemma for the domain $\Omega=[0, \ell] \times[0,1]$ as we do in Section 4 , by conformal invariance of the $\mathrm{SLE}_{\kappa}$.

LEMMA A.1. Suppose $\mathcal{E}$ is a random subset of $\partial \mathbb{U}$ satisfying the following: there are constants $C>0$ and $\beta \in(0,1)$ such that, for any interval $I \subset \partial \mathbb{U}$, we have

$$
\mathbb{P}[\mathcal{E} \cap I \neq \varnothing] \leq C|I|^{\beta} .
$$

Then, for any subset $A \subset \partial \mathbb{U}$, the following hold:

1. If $\operatorname{dim}(A)<\beta$, then

$$
A \cap \mathcal{E}=\varnothing, \quad \text { almost surely. }
$$

2. If $\operatorname{dim}(A) \geq \beta$, then

$$
\operatorname{dim}(A \cap \mathcal{E}) \leq \operatorname{dim}(A)-\beta, \quad \text { almost surely. }
$$

This lemma is a part of [33], Lemma 2.3, where the authors give a more complete description of the set $A \cap \mathcal{E}$. The above cases are sufficient to our purposes in the proof of Proposition 4.9, so we include their proofs in this Appendix.

Proof of ITEM 1. Since $\beta>\operatorname{dim}(A)$, for any $\varepsilon>0$, there exists a cover $\bigcup_{i} I_{i}$ of $A$ such that $\sum_{i}\left|I_{i}\right|^{\beta} \leq \varepsilon$. Therefore,

$$
\mathbb{P}[A \cap \mathcal{E} \neq \varnothing] \leq \sum_{i} \mathbb{P}\left[I_{i} \cap \mathcal{E} \neq \varnothing\right] \leq C \sum_{i}\left|I_{i}\right|^{\beta} \leq C \varepsilon
$$

almost surely. Letting $\varepsilon \rightarrow 0$, we see that $\mathbb{P}[A \cap \mathcal{E} \neq \varnothing]=0$.

ProOf OF ITEM 2. For any $\gamma>\operatorname{dim}(A)-\beta$, there exists a cover $\bigcup_{i} I_{i}$ of $A$ such that $\sum_{i}\left|I_{i}\right|^{\beta+\gamma}<\infty$. Hence, we have

$$
\mathbb{E}\left[\sum_{i}\left|I_{i}\right|^{\gamma} \mathbb{1}_{\left\{I_{i} \cap \mathcal{E} \neq \varnothing\right\}}\right]=\sum_{i}\left|I_{i}\right|^{\gamma} \mathbb{P}\left[I_{i} \cap \mathcal{E} \neq \varnothing\right] \leq C \sum_{i}\left|I_{i}\right|^{\beta+\gamma}<\infty,
$$

almost surely. Thus, the collection $\left\{I_{i}: I_{i} \cap \mathcal{E} \neq \varnothing\right\}$ is a cover of $A \cap \mathcal{E}$ and $\sum_{i}\left|I_{i}\right|^{\gamma} \times$ $1_{\left\{I_{i} \cap \mathcal{E} \neq \varnothing\right\}}<\infty$, almost surely. Therefore, we have

$$
\operatorname{dim}(A \cap \mathcal{E}) \leq \gamma, \quad \text { almost surely. }
$$

As this holds for any $\gamma>\operatorname{dim}(A)-\beta$, we have $\operatorname{dim}(A \cap \mathcal{E}) \leq \operatorname{dim}(A)-\beta$, almost surely.

Acknowledgments. We thank C. Garban, K. Izyurov, A. Karrila, A. Kemppainen and F. Viklund for interesting and useful discussions. We thank I. Manolescu for very useful comments on the RSW estimates. We also thank the referee for careful comments which improved the presentation, provided an alternative proof for the existence of the global 2SLEs in Section 3, and clarified Section 4.

V. Beffara is funded by the ANR project ANR-16-CE40-0016. E. Peltola is funded by the Deutsche Forschungsgemeinschaft (DFG, German Research Foundation) under Germany's Excellence Strategy-EXC-2047/1-390685813. H. Wu is funded by Beijing Natural Science Foundation (JQ20001, Z180003) and Chinese Thousand Talents Plan for Young Professionals. During this work, E. Peltola and H. Wu were supported by the ERC AG COMPASP, the NCCR SwissMAP, and the Swiss NSF. Part of this work was done while E. Peltola and H. $\mathrm{Wu}$ visited the IHES, and the first version of the paper was completed while E. Peltola and $\mathrm{H}$. Wu were visiting the MFO as a "research pair." 


\section{REFERENCES}

[1] Aizenman, M. and Burchard, A. (1999). Hölder regularity and dimension bounds for random curves. Duke Math. J. 99 419-453. MR1712629 https://doi.org/10.1215/S0012-7094-99-09914-3

[2] Alberts, T. and Kozdron, M. J. (2008). Intersection probabilities for a chordal SLE path and a semicircle. Electron. Commun. Probab. 13 448-460. MR2430712 https://doi.org/10.1214/ECP.v13-1399

[3] Bauer, M., Bernard, D. and Kytölä, K. (2005). Multiple Schramm-Loewner evolutions and statistical mechanics martingales. J. Stat. Phys. 120 1125-1163. MR2187598 https://doi.org/10.1007/ s10955-005-7002-5

[4] Camia, F. and Newman, C. M. (2007). Critical percolation exploration path and $S L E_{6}$ : A proof of convergence. Probab. Theory Related Fields 139 473-519. MR2322705 https://doi.org/10.1007/ s00440-006-0049-7

[5] Chelkak, D., Duminil-Copin, H., Hongler, C., Kemppainen, A. and Smirnov, S. (2014). Convergence of Ising interfaces to Schramm's SLE curves. C. R. Math. Acad. Sci. Paris 352 157-161. MR3151886 https://doi.org/10.1016/j.crma.2013.12.002

[6] Chelkak, D., Hongler, C. and Izyurov, K. (2015). Conformal invariance of spin correlations in the planar Ising model. Ann. of Math. (2) 181 1087-1138. MR3296821 https://doi.org/10.4007/annals. 2015.181.3.5

[7] Chelkak, D. and Smirnov, S. (2012). Universality in the 2D Ising model and conformal invariance of fermionic observables. Invent. Math. 189 515-580. MR2957303 https://doi.org/10.1007/ s00222-011-0371-2

[8] Dubédat, J. (2007). Commutation relations for Schramm-Loewner evolutions. Comm. Pure Appl. Math. 60 1792-1847. MR2358649 https://doi.org/10.1002/cpa.20191

[9] DubÉdAT, J. (2009). Duality of Schramm-Loewner evolutions. Ann. Sci. Éc. Norm. Supér. (4) 42 697-724. MR2571956 https://doi.org/10.24033/asens.2107

[10] Duminil-Copin, H., Sidoravicius, V. and Tassion, V. (2017). Continuity of the phase transition for planar random-cluster and Potts models with $1 \leq q \leq 4$. Comm. Math. Phys. 349 47-107. MR3592746 https://doi.org/10.1007/s00220-016-2759-8

[11] Flores, S. M. and Kleban, P. (2015). A solution space for a system of null-state partial differential equations: Part 4. Comm. Math. Phys. 333 669-715. MR3296160 https://doi.org/10.1007/ s00220-014-2180-0

[12] Garban, C. and WU, H. (2020). On the convergence of FK-Ising percolation to $S L E(16 / 3,(16 / 3)-6)$. J. Theoret. Probab. 33 828-865. MR4091584 https://doi.org/10.1007/s10959-019-00950-9

[13] Graham, K. (2007). On multiple Schramm-Loewner evolutions. J. Stat. Mech. Theory Exp. 3 P03008. MR2318432 https://doi.org/10.1088/1742-5468/2007/03/p03008

[14] Hongler, C. and Smirnov, S. (2013). The energy density in the planar Ising model. Acta Math. 211 191-225. MR3143889 https://doi.org/10.1007/s11511-013-0102-1

[15] Izyurov, K. (2017). Critical Ising interfaces in multiply-connected domains. Probab. Theory Related Fields 167 379-415. MR3602850 https://doi.org/10.1007/s00440-015-0685-x

[16] KARRILA, A. (2018). Limits of conformal images and conformal images of limits for planar random curves. Available at arXiv: 1810.05608.

[17] Karrila, A. (2019). Multiple SLE type scaling limits: From local to global. Available at arXiv:1903.10354.

[18] Karrila, A., Kytölä, K. and Peltola, E. (2020). Boundary correlations in planar LERW and UST. Comm. Math. Phys. 376 2065-2145. MR4104543 https://doi.org/10.1007/s00220-019-03615-0

[19] Kemppainen, A. and Smirnov, S. (2017). Random curves, scaling limits and Loewner evolutions. Ann. Probab. 45 698-779. MR3630286 https://doi.org/10.1214/15-AOP1074

[20] Kemprainen, A. and SMIRnov, S. (2018). Configurations of FK Ising interfaces and hypergeometric SLE. Math. Res. Lett. 25 875-889. MR3847338 https://doi.org/10.4310/MRL.2018.v25.n3.a7

[21] Kozdron, M. J. and LAWLER, G. F. (2007). The configurational measure on mutually avoiding SLE paths. In Universality and Renormalization. Fields Inst. Commun. 50 199-224. Amer. Math. Soc., Providence, RI. MR2310306 https://doi.org/10.1088/1751-8113/45/49/494015

[22] Kytölä, K. and Peltola, E. (2016). Pure partition functions of multiple SLEs. Comm. Math. Phys. 346 237-292. MR3528421 https://doi.org/10.1007/s00220-016-2655-2

[23] Lawler, G., Schramm, O. and Werner, W. (2003). Conformal restriction: The chordal case. J. Amer. Math. Soc. 16 917-955. MR1992830 https://doi.org/10.1090/S0894-0347-03-00430-2

[24] Lawler, G. F. (2005). Conformally Invariant Processes in the Plane. Mathematical Surveys and Monographs 114. Amer. Math. Soc., Providence, RI. MR2129588 https://doi.org/10.1090/surv/114

[25] LAWLER, G. F. (2009). Partition functions, loop measure, and versions of SLE. J. Stat. Phys. 134 813-837. MR2518970 https://doi.org/10.1007/s10955-009-9704-6 
[26] Lawler, G. F., Schramm, O. and Werner, W. (2004). Conformal invariance of planar loop-erased random walks and uniform spanning trees. Ann. Probab. 32 939-995. MR2044671 https://doi.org/10. 1214/aop/1079021469

[27] Lawler, G. F. and Werner, W. (2004). The Brownian loop soup. Probab. Theory Related Fields 128 565-588. MR2045953 https://doi.org/10.1007/s00440-003-0319-6

[28] Miller, J. and Sheffield, S. (2016). Imaginary geometry II: Reversibility of $\operatorname{S} L E_{\kappa}\left(\rho_{1} ; \rho_{2}\right)$ for $\kappa \in$ (0, 4). Ann. Probab. 44 1647-1722. MR3502592 https://doi.org/10.1214/14-AOP943

[29] Miller, J. and ShefField, S. (2016). Imaginary geometry I: Interacting SLEs. Probab. Theory Related Fields 164 553-705. MR3477777 https://doi.org/10.1007/s00440-016-0698-0

[30] Miller, J., ShefField, S. and Werner, W. (2020). Non-simple SLE curves are not determined by their range. J. Eur. Math. Soc. (JEMS) 22 669-716. MR4055986 https://doi.org/10.4171/jems/930

[31] Peltola, E. and Wu, H. (2019). Global and local multiple SLEs for $\kappa \leq 4$ and connection probabilities for level lines of GFF. Comm. Math. Phys. 366 469-536. MR3922531 https://doi.org/10.1007/ s00220-019-03360-4

[32] Rohde, S. and Schramm, O. (2005). Basic properties of SLE. Ann. of Math. (2) 161 883-924. MR2153402 https://doi.org/10.4007/annals.2005.161.883

[33] Rohde, S. and WU, H. (In preparation). On the dimension of the SLE trace in simply connected domains.

[34] Schramm, O. (2000). Scaling limits of loop-erased random walks and uniform spanning trees. Israel J. Math. 118 221-288. MR1776084 https://doi.org/10.1007/BF02803524

[35] SCHRAmm, O. (2007). Conformally invariant scaling limits: An overview and a collection of problems. In International Congress of Mathematicians. Vol. I 513-543. Eur. Math. Soc., Zürich. MR2334202 https://doi.org/10.4171/022-1/20

[36] Schramm, O. and ShefField, S. (2009). Contour lines of the two-dimensional discrete Gaussian free field. Acta Math. 202 21-137. MR2486487 https://doi.org/10.1007/s11511-009-0034-y

[37] ShefField, S. and WeRner, W. (2012). Conformal loop ensembles: The Markovian characterization and the loop-soup construction. Ann. of Math. (2) 176 1827-1917. MR2979861 https://doi.org/10.4007/ annals.2012.176.3.8

[38] Smirnov, S. (2001). Critical percolation in the plane: Conformal invariance, Cardy's formula, scaling limits. C. R. Acad. Sci. Paris Sér. I Math. 333 239-244. MR1851632 https://doi.org/10.1016/ S0764-4442(01)01991-7

[39] SMIRnov, S. (2006). Towards conformal invariance of 2D lattice models. In International Congress of Mathematicians. Vol. II 1421-1451. Eur. Math. Soc., Zürich. MR2275653

[40] SMIRnov, S. (2010). Conformal invariance in random cluster models. I. Holomorphic fermions in the Ising model. Ann. of Math. (2) 172 1435-1467. MR2680496 https://doi.org/10.4007/annals.2010.172.1441

[41] Werner, W. (2005). Conformal restriction and related questions. Probab. Surv. 2 145-190. MR2178043 https://doi.org/10.1214/154957805100000113

[42] Werner, W. and Wu, H. (2013). From CLE( $\kappa)$ to SLE( $\kappa, \rho)$ 's. Electron. J. Probab. 18 36. MR3035764 https://doi.org/10.1214/EJP.v18-2376

[43] WU, H. (2020). Hypergeometric SLE: Conformal Markov characterization and applications. Comm. Math. Phys. 374 433-484. MR4072221 https://doi.org/10.1007/s00220-020-03697-1 